ARTICLE

Received 4 Aug 2014 | Accepted 12 Jan 2015 | Published 9 Feb 2015

DOI: $10.1038 /$ ncomms7289

\title{
A leak pathway for luminal protons in endosomes drives oncogenic signalling in glioblastoma
}

\author{
Kalyan C. Kondapalli ${ }^{1, *, \dagger}$, Jose P. Llongueras ${ }^{1, \star}$, Vivian Capilla-González ${ }^{2, \dagger}$, Hari Prasad ${ }^{1}$, Anniesha Hack ${ }^{1}$, \\ Christopher Smith², Hugo Guerrero-Cázares², Alfredo Quiñones-Hinojosa² \& Rajini Rao
}

Epidermal growth factor receptor (EGFR) signalling is a potent driver of glioblastoma, a malignant and lethal form of brain cancer. Disappointingly, inhibitors targeting receptor tyrosine kinase activity are not clinically effective and EGFR persists on the plasma membrane to maintain tumour growth and invasiveness. Here we show that endolysosomal $\mathrm{pH}$ is critical for receptor sorting and turnover. By functioning as a leak pathway for protons, the $\mathrm{Na}^{+} / \mathrm{H}^{+}$ exchanger NHE9 limits luminal acidification to circumvent EGFR turnover and prolong downstream signalling pathways that drive tumour growth and migration. In glioblastoma, NHE9 expression is associated with stem/progenitor characteristics, radiochemoresistance, poor prognosis and invasive growth in vitro and in vivo. Silencing or inhibition of NHE9 in brain tumour-initiating cells attenuates tumoursphere formation and improves efficacy of EGFR inhibitor. Thus, NHE9 mediates inside-out control of oncogenic signalling and is a highly druggable target for pan-specific receptor clearance in cancer therapy.

\footnotetext{
${ }^{1}$ Department of Physiology, The Johns Hopkins University School of Medicine, 725 North Wolfe Street, Baltimore, Maryland 21205, USA. ${ }^{2}$ Department of Neurosurgery, The Johns Hopkins University School of Medicine, 725 North Wolfe Street, Baltimore, Maryland 21205, USA. ${ }^{\star}$ These authors contributed equally to this work. †Present addresses: Department of Natural Sciences, University of Michigan-Dearborn, 4901 Evergreen Road, Dearborn, Michigan 48128, USA (K.C.K.); Department of Stem Cells, Andalusian Center for Molecular Biology and Regenerative Medicine (CABIMER), Américo Vespucio, s/n, 41092 Seville, Spain (V.C.-G.). Correspondence and requests for materials should be addressed to A.Q.-H. (email: aquinon2@jhmi.edu) or to R.R. (email: rrao@jhmi.edu).
} 
$\mathrm{G}$ lioblastoma multiforme (GBM) is the most common and aggressive form of adult primary brain tumour with extremely poor prognosis ${ }^{1,2}$. Diffuse invasion of tumour cells into the surrounding brain parenchyma makes surgical resection largely ineffective and the pluripotent behaviour of a small group of brain tumour-initiating cells (BTICs) in GBM confers drug resistance and recurrence even after aggressive treatment ${ }^{3-5}$. As a result, clinical outcomes of patients have failed to show substantive improvement over median survival times of 12 months and innovative approaches to therapy are urgently needed $^{5}$. The key step in moving towards personalized medicine involves identifying specific genes and pathways to be targeted in individual patients. This has been the driving force for compiling databases of genome and proteome changes in GBM patients that are now being successfully mined to uncover novel risk factors and potential therapeutic targets. Using this approach, we made the unexpected discovery that in a subset of glioblastomas, the autism-associated gene SLC9A9 (refs 6-8) was a potent driver of tumour growth and migration, associated with decreased patient survival and increased aggressiveness in vivo.

Previously, we showed that loss-of-function mutations in SLC9A9 encoding the endosomal $\mathrm{Na}^{+} / \mathrm{H}^{+}$exchanger NHE9 result in hyperacidification of sorting endosomes and cellular trafficking defects associated with autism ${ }^{6,7}$. In contrast, we now report that gain of function in NHE9 results in excessive luminal alkalization in BITCs from patient glioblastomas. Although studies of $\mathrm{pH}$, ionic and volume modulation in tumour cells have thus far been restricted to plasma membrane ion transporters, there have been intriguing hints of a link between organellar $\mathrm{pH}$ and oncogenic signalling by growth factor receptors. The human papillomavirus type 16 E5 oncoprotein activates growth factor receptors Epidermal growth factor receptor (EGFR) and platelet-derived growth factor receptor with concomitant alkalization of Golgi and endosomes, following binding to $\mathrm{H}^{+}$-pumping $\mathrm{V}$-ATPase $16 \mathrm{kDa} \quad c$ subunit ${ }^{9}$. Furthermore, pharmacological disruption of V-ATPase activity by bafilomycin $A_{1}$ was found to augment the extent and duration of EGFR activation ${ }^{10}$. However, a causal link between luminal $\mathrm{pH}$ and oncogenic signalling remains to be established, and the prevailing model of the luminal $\mathrm{pH}$ set point overwhelmingly focuses on the proton pumping V-ATPase, with the role of proton leak pathways, mediated by endosomal $\mathrm{Na}^{+} / \mathrm{H}^{+}$ exchange, being largely understudied.

In this study we demonstrate that NHE9 levels in patientderived BTICs drive proliferation rates, cell adhesion, migration directionality and speed in vitro and in vivo mouse xenograft models. Mechanistically, these events are mediated by the luminal $\mathrm{pH}$ of sorting endosomes with distinct effects on endocytosis and exocytosis of cargo. EGFR is mutated, amplified or transcriptionally upregulated in $50 \%$ of human $\mathrm{GBM}^{11}$ and its downstream signalling network includes highly deregulated and oncogenic components responsible for uncontrolled cell proliferation in most cancers. By drastically attenuating receptor turnover, NHE9 exerts posttranslational control on membrane persistence of EGFR, confers increased resistance to receptor tyrosine kinase inhibitors and drives oncogenic signalling pathways that regulate cell division and migration. Importantly, posttranslational control by NHE9 overrides differences in EGFR transcript levels in GBM tumour cells. Finally, we demonstrate that a pharmacological inhibitor of NHE transporters works together with a known EGFR inhibitor to impair GBM tumour growth. Given the dismal prognosis of glioblastoma, these observations highlight the potential of developing selective anti-NHE9 therapeutics. Taken together, our findings reveal a crucial, hitherto unrecognized role for luminal $\mathrm{pH}$ in GBM progression and allow us to propose NHE9 as a promising chemotherapeutic target.

\section{Results}

NHE9 affects patient survival and GBM proliferation. Gene expression profiles for NHE9 (SLC9A9) were extracted from human glioblastoma arrays on Oncomine and The Cancer Genome Atlas (TCGA) databases ${ }^{12-16}$. NHE9 expression varied widely among human glioblastoma subtypes, with $36 \%$ of mesenchymal tumours showing significant NHE9 upregulation (Supplementary Fig. 1A). Notably, SLC9A9 was in the top $12 \%$ of overexpressed genes in a human gene chip array of 101 samples, with messenger RNA levels averaging 5.7-fold higher $(P=0.012$; Student's $t$-test) in glioblastomas relative to control neural stem cells (Fig. 1a). Interrogation of the TCGA database revealed that patients with unaltered expression of NHE9 $(71.4 \%$ of 91 GBM cases) survived longer with the difference in maximal survival between these patients of 65 months (Fig. 1b; $P=0.032$; log-rank test). In addition, patients with unaltered expression of NHE9 remained disease free for a median period of 8.4 months after tumour resection. In contrast, NHE9 upregulation $(28.6 \%$ of 91 GBM cases) correlated with significantly shorter disease-free periods with a median of only 4.8 months (Supplementary Fig. 1B; $P=0.012$; log-rank test). A more thorough examination of the TCGA data set including a subset of patients who received neoadjuvant chemotherapy, which included temozolomide ( $>50 \%$ cases), PCV (procarbazine, CCNU and vincristine) and others, revealed that patients with unaltered expression of NHE9 showed strikingly better response and survived longer with a median survival of 58.68 months, in contrast to 15.98 months in patients with NHE9 upregulation (Fig. 1c; $P=0.000002$; log-rank test). Furthermore, patients with unaltered expression of NHE9 with neoadjuvant chemotherapy remained disease free after resection for a median period of 33.67 months, in comparison with 11.28 months in patients with NHE9 upregulation (Supplementary Fig. 1C; $P=0.00295$; log-rank test). The finding that NHE9 confers resistance to treatment is strengthened by a recent study demonstrating that patients with oesophageal squamous cell carcinomas (ESCCs) with NHE9 upregulation showed poor response to neoadjuvant chemoradiotherapy ${ }^{17}$. Taken together, the observations suggest that NHE9 expression levels may have a role in GBM prognosis.

Based on these initial observations, we evaluated NHE9 transcript levels in three clinical GBM specimens (GBM 612, GBM 253 and GBM 276; Supplementary Table 1), cultured in serum-free conditions to enrich for BTIC that maintain phenotype and genotype characteristics of the original tumour. NHE9 expression, similar to that of CD133 (ref. 15), was associated with stem/progenitor characteristics, as seen by a significant decrease in transcript on culturing GBM 612 in serum (Fig. 1d. No transcriptional change was observed for the closely related isoform NHE6 after transfer to serum. Comparative analysis of three early-passage BTIC lines derived from patient tumours showed a 19.4-fold variation in NHE9 transcript levels (GBM $276<$ GBM $253<$ GBM 612; Fig. 1e), consistent with the variation observed in database surveys. The correlation of NHE9 levels with higher growth rates in GBM 612 relative to GBM 276 was suggestive of a role in tumour cell proliferation (Fig. 1f). To test this hypothesis, we engineered lentiviral-mediated elevation of NHE9 in GBM 276 and, conversely, attenuated NHE9 transcripts in GBM 612 as shown Fig. 1g. No compensatory changes in mRNA levels were observed in the related endosomal isoform NHE6 or V-ATPase subunit $V_{O} A_{1}$ in these cells (Fig. 1g). Consistent with a role for NHE9 in tumour cell proliferation, growth rates in GBM 276 cells overexpressing NHE9 were significantly higher than vector-transfected control cells (Fig. 1h). Conversely, knocking down NHE9 expression decreased cell proliferation in GBM 612 cells (Fig. 1i). These findings were replicated under both normoxia and hypoxia conditions 
(Supplementary Fig. 2A-D). We note that cell viability remained unaltered by introduction or knockdown of NHE9 (Supplementary Fig. 2E). Therefore, we conclude that NHE9 levels regulate proliferation of BTIC in vitro.

NHE9 promotes GBM tumorigenesis and invasion. Next, we evaluated in vivo effects of widely differing NHE9 expression in two human glioblastomas, GBM 276 (Fig. 2a,b) and GBM 612 (Fig. 2c,d), using intracranial xenograft transplants in a nude mouse model (Fig. 2e). Following silencing of NHE9 in GBM 612 (GBM 612 NHE9-), tumour area in implants was visibly reduced relative to vector-transfected control (Fig. 2f,g). These differences were quantitatively significant by an average of $\sim 2.2$-fold (Fig. 2h; $P=5.3 \times 10^{-6}$; two-tailed Welch's $t$-test). Overexpression of NHE9 in GBM 276 (GBM 276 NHE9+ ) resulted in a significant increase in tumour size $(\sim 2$-fold on average; $P=6.1 \times 10^{-5}$; two-tailed Welch's $t$-test) relative to the vectortransfected control (Fig. 2i and Supplementary Fig. 3). Invasive capacity was assessed from the number of cells migrating into the contralateral hemisphere, as described previously ${ }^{2}$. Similar to the observations with tumour size, there was a significant reduction of tumour cell invasion following NHE9 silencing in GBM 612 $(P=0.0025$; two-tailed Welch's $t$-test $)$ and increased invasion with NHE9 overexpression in GBM $276(P=0.0003$; two-tailed Welch's $t$-test; Fig. $2 \mathrm{j}, \mathrm{k}$ ) when we analysed cell migration in vivo. a

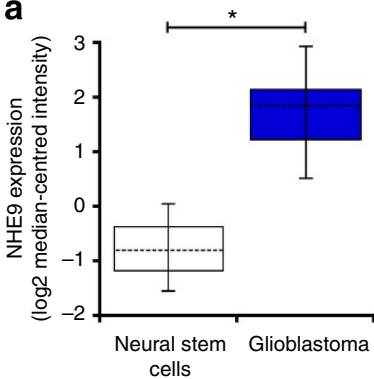

d

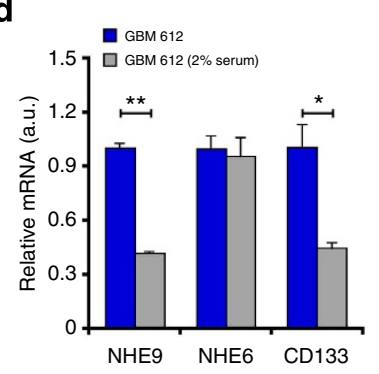

g

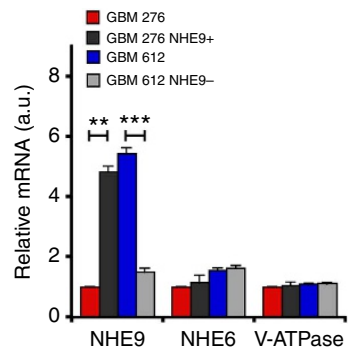

b

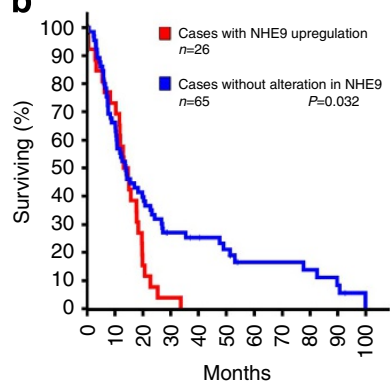

e

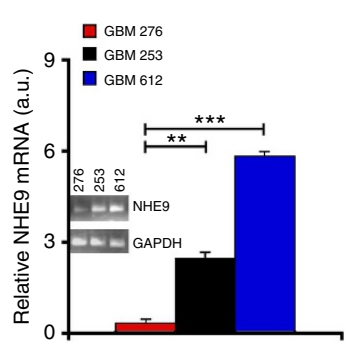

h

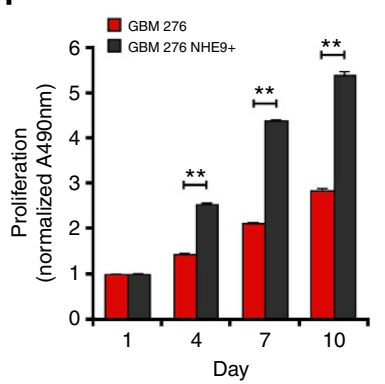

C

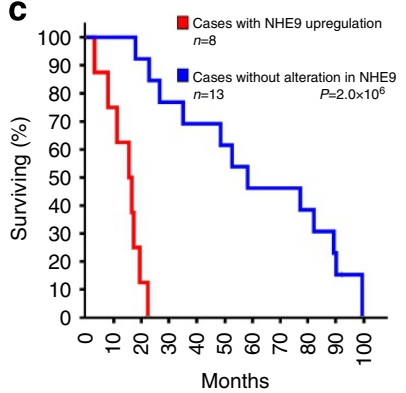

f

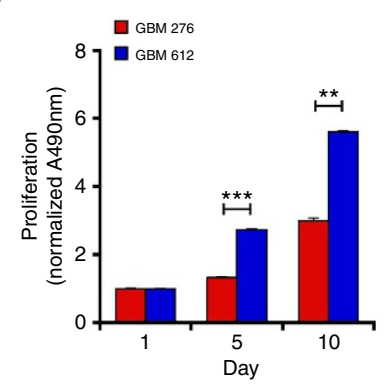

i

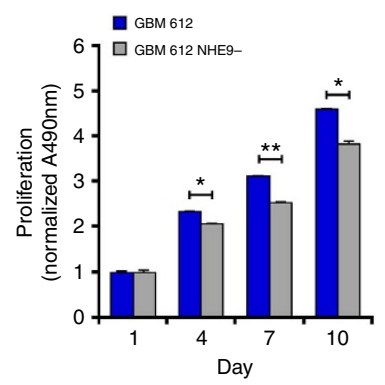

Figure 1 | NHE9 expression in glioblastoma affects patient survival, chemoradiation resistance and tumour stem cell proliferation. (a) NHE9 transcript is elevated in glioblastoma tumour stem cells $(n=22)$ relative to normal neural stem cells $(n=3)\left({ }^{\star} P=0.012\right.$, Student's $t$-test). (b-c) NHE9 transcript is upregulated in $28.6 \%$ of cases found in TCGA database when a Z-score of 0.5 was selected. (b) Patients with GBM upregulation of NHE9 had decreased overall survival. (c) Of 21 patients who received neoadjuvant chemoradiotherapy, those with unaltered expression of NHE9 showed better response to treatment and survived longer ( $P=0.000002$; log-rank test) relative to those with upregulation of NHE9. (d) Quantitative PCR ( $9 P C R$ ) analysis of NHE9, NHE6 and CD133 mRNA from GBM 612 tumour stem cells cultured in medium without or with serum. Transcript levels of NHE9 and CD133 decrease in serum ( ${ }^{\star} P=0.012,{ }^{\star \star} P=0.0001$, two-tailed Student's $t$-test with Welch's correction, $n=3$ ), whereas NHE6 shows no change. (e) qPCR analysis of NHE9 mRNA in cells from three primary GBM tumours 276,253 and $612\left({ }^{\star \star} P=0.0003\right.$, ${ }^{\star \star \star} P=1.01 \times 10^{-5}$, two-tailed Welch's $t$-test, $\left.n=3\right)$. Inset shows ethidium bromide-stained agarose gel of reverse transcriptase-PCR products of mRNA from the GBM cells. (f) Normalized proliferation of GBM cells 276 and $612\left({ }^{\star \star \star} P=1.87 \times 10^{-5},{ }^{\star \star} P=0.0001\right.$, two-tailed Welch's $t$-test, $\left.n=3\right)$ as determined by MTS assay. (g) qPCR analysis showing the efficacy of overexpression and shRNA knockdown of NHE9 in GBM 276 and 612 cells, respectively ( ${ }^{\star \star} P=0.0008$, ${ }^{\star \star \star} P=3.44 \times 10^{-5}$, two-tailed Welch's $t$-test, $n=3$ ). (h) Normalized proliferation of GBM 276 control cells and 276 cells overexpressing NHE9 (**P<0.01, two-tailed Welch's $t$-test, $n=3$ ).

(i) Normalized proliferation of GBM 612 control cells and 612 cells with shRNA knockdown of NHE9 ( ${ }^{\star} P<0.05$, ${ }^{\star \star} P=0.0018$, two-tailed Welch's $t$-test, $n=3)$. Bars represent mean \pm s.d. All cell cultures were counted, seeded at similar levels and cell densities determined by measuring absorbance at $490 \mathrm{~nm}$ (Abs490 nm); initial values were as follows (average of triplicates, s.d.) GBM 276 (0.148, 0.010); GBM 276 + NHE9 (0.155, 0.011$)$; GBM 612 (0.174, 0.022); GBM 612-NHE9 (0.167, 0.041). See related Supplementary Figs 1 and 2. 

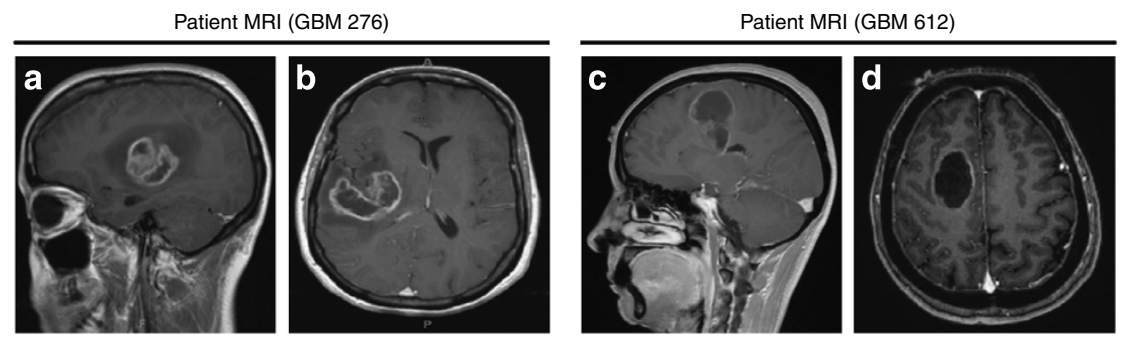

e
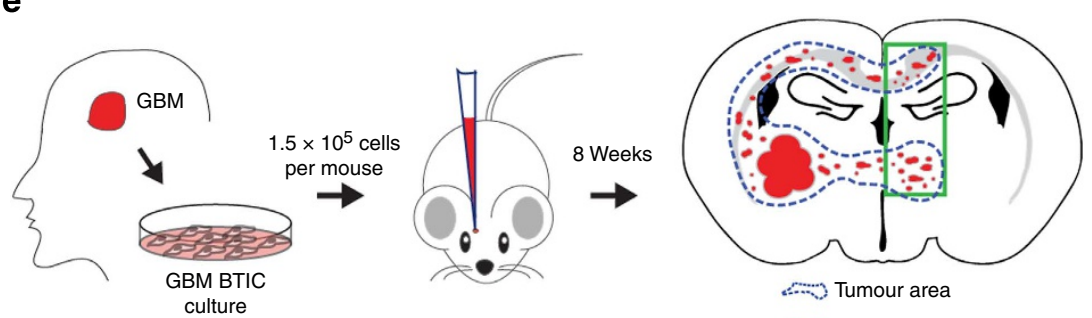

Tumour area

$\square$ Migrating cells
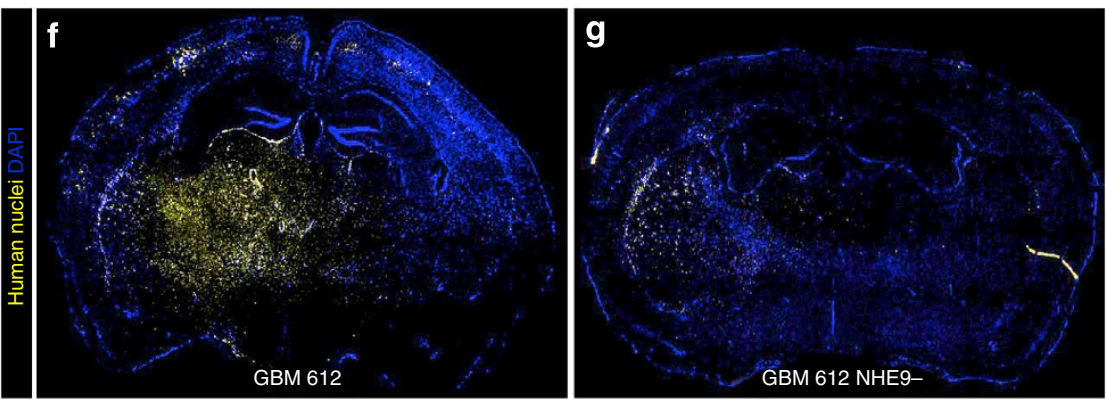

h

i
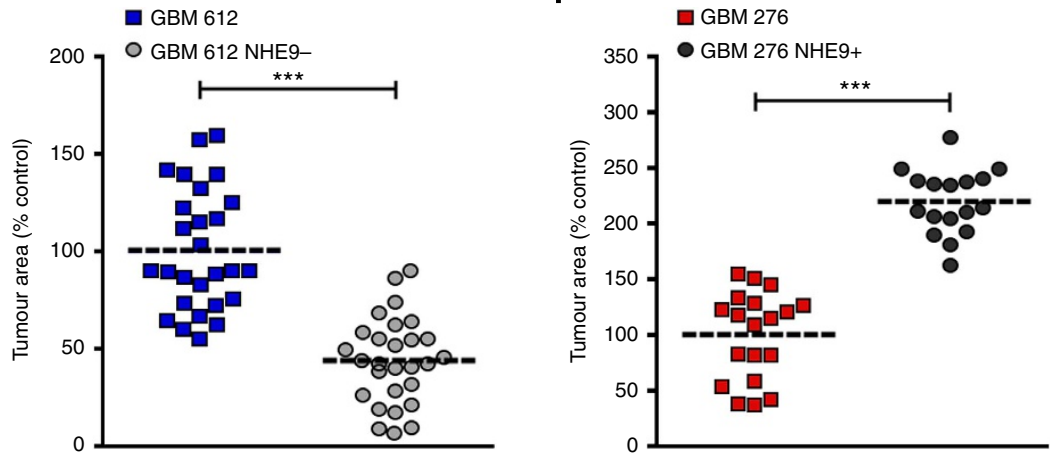

j

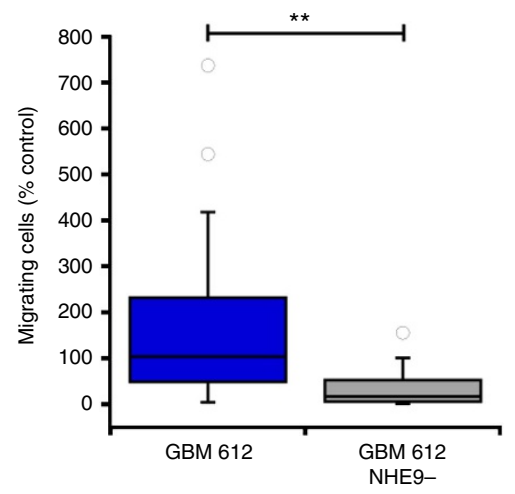

k

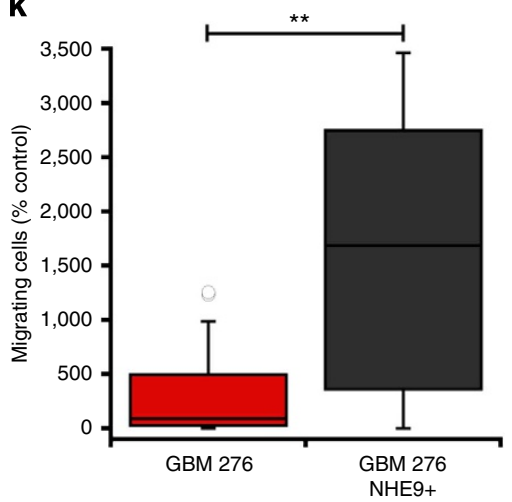


Together, both in vitro and in vivo findings confirm a critical role for NHE9 in tumour progression.

NHE9 drives GBM BTIC migration. The diffuse and aggressive infiltration of tumour cells along vascular and white matter tracts is directly responsible for the inefficacy of tumour resection, resulting in mean survival times of only 12-15 months. Central to the invasiveness associated with glioblastomas, as observed in Fig. 2, are tumour cell-substratum interactions and subsequent modification of matrix barriers. In vivo, tumour cells interact with the extracellular matrix in the nanometre scale ${ }^{18}$ and respond to topographical cues that affect cell adhesion and gene expression. To study BTIC migration in vitro under conditions of GBM dissemination ${ }^{2,18-20}$, we conducted directional migration assays on nano-ridges constructed of transparent polyurethane acrylate coated with laminin (Fig. 3a) as previously reported by our group ${ }^{2}$. These longitudinal tracts closely mimic mechanical cues found in extracellular matrix such as capillaries and nerve fibre tracts along which tumour cells migrate. Biased migration along the grooves was quantified using time-lapse microscopy for a period of $15 \mathrm{~h}$ at regular intervals of $15 \mathrm{~min}$ in GBM pairs 276 and 612, with NHE9 overexpressed or silenced, respectively. Phase-contrast images of a representative 2 -h period for GBM 276 and GBM 276 NHE9 + is shown in Fig. 3b. Quantification of cell speed over time for each population (Fig. $3 c, d$ ) and the total average cell speed (Fig. $3 \mathrm{e}-\mathrm{g}$ ) revealed that elevation of NHE9 expression in GBM 276 increased migration speed and consequently distance travelled, whereas attenuation of the high expression of NHE9 in GBM 612 had the converse effect, consistent with the invasion trends observed in mouse xenografts. Next, we evaluated specific parameters of migratory behaviour likely to contribute to invasive ability. Migration alignment, which is the ratio of cell movement parallel to the patterned ridges to that perpendicular to the pattern (Fig. $3 \mathrm{~h}$ ), correlated with migration speed and a significant change in alignment was observed on attenuation of NHE9 in GBM 612 cells (Fig. 3i,j). Migration persistence, ratio of the actual distance travelled by the cell to the shortest distance between the initial and final points (Fig. 3k), was also significantly affected by changes in NHE9 expression in both the GBM lines (Fig. 31,m). Thus, multiple parameters confirm that NHE9 regulates tumour cell migration in brain cancer.

NHE9 modulates endosomal $\mathbf{p H}$ and trafficking. As a starting point to decipher the mechanistic basis for tumour promotion, we focused on GBM 253, in which NHE9 expression was intermediate between GBM 276 and GBM 612 BTIC (Fig. 1e). Ectopic expression of NHE9 in GBM 253 significantly increased cell proliferation (Supplementary Fig. 4A), consistent with results from Fig. 1. We showed that NHE9-GFP partly co-localized with EEA1 (Supplementary Fig. 5A,B) and Rab 11 (Fig. 4a,b), markers for early and recycling endosomes, respectively, in GBM 253. We did not observe co-localization of NHE9-GFP with lysobisphosphatidic acid, a marker for late endosomes (Supplementary Fig. 5C,D). Lack of co-localization with endoplasmic reticulum marker and protein-folding chaperone $\mathrm{BiP}$ suggested that NHE9 protein processing was not defective in GBM (Supplementary Fig. 5E,F). Therefore, we concluded that NHE9 localization in tumour cells was similar to that observed in normal, primary murine astrocytes ${ }^{6}$. Furthermore, NHE9-GFP co-localized with endocytosed transferrin tagged to Alexa Fluor633 , both in the perinuclear region and in the periphery of the cell (Fig. 4c), also seen in orthogonal section (Fig. 4d). We monitored steady-state accumulation of transferrin (Fig. 4e) and noted that NHE9-GFP expression in GBM 253 cells was accompanied by increased accumulation of Tfn-Alexa Fluor 633, relative to green fluorescent protein (GFP)-transfected controls, as measured by flow cytometry (Fig. 4f,g) and confocal microscopy (Fig. 4h,i). To determine whether increased transferrin accumulation (Fig. 4j) was dependent on $\mathrm{Na}^{+} / \mathrm{H}^{+}$exchange activity and not a nonspecific effect of gene overexpression, we evaluated the effect of previously described autism-associated loss-of-function mutations in NHE9 (refs 6,8). Single amino-acid substitutions in conserved regions of mammalian NHE9, L236S and S438P (Fig. 4l) were generated and introduced into GBM 253 cells. Mutant NHE9 (S438P) failed to increase cell proliferation, in contrast to wild-type control (Supplementary Fig. 4B). Similar to the wild-type control, loss-of-function NHE9 mutants also colocalized with Tfn-Alexa Fluor 633 (Supplementary Fig. 5G,H), but failed to elevate Tfn-Alexa Fluor 633 accumulation (Fig. 4k). Therefore, we concluded that ion transport activity by NHE9 alters transferrin trafficking in GBM cells.

NHE9 is proposed to function as a leak pathway for protons, limiting the acidification of the endosomal lumen by the proton pumping V-ATPase ${ }^{6}$. Therefore, high levels of NHE9 should make the endosomal lumen more alkaline, via cation/proton exchange activity. To measure endosomal $\mathrm{pH}\left(\mathrm{pH}_{\mathrm{e}}\right)$, we took advantage of co-localization of NHE9 with transferrin, as shown in Fig. 4c,d. Luminal $\mathrm{pH}$ was quantified by a ratiometric method using $\mathrm{pH}$-sensitive fluorescence of fluorescein isothiocyanate (FITC)-tagged transferrin, normalized for differences in endosomal uptake with $\mathrm{pH}$-insensitive fluorescence of Alexa Fluor-633 linked to transferrin (Fig. 5a) and calibrated using buffers of known pH (Fig. 5b). Expression of NHE9 in GBM 253 cells resulted in $>1 \mathrm{pH}$ unit elevation of $\mathrm{pH}_{\mathrm{e}}$ from $5.71 \pm 0.23$ to $6.90 \pm 0.47$ (Fig. 5c). In contrast, there was no significant change in cytoplasmic $\mathrm{pH}$ (Supplementary Fig. 4C,D), as has been previously reported for the closely related endosomal isoform NHE6 (ref. 21). To establish a causal link between compartmental $\mathrm{pH}$ and trafficking of transferrin, we evaluated a known alkalinizing agent, monensin, which also mediates $\mathrm{Na}^{+} / \mathrm{H}^{+}$ exchange activity. Luminal $\mathrm{pH}_{\mathrm{e}}$ in GBM 253 cells treated with monensin $(500 \mu \mathrm{M})$ was also elevated (to $7.02 \pm 0.20)$, similar to cells expressing NHE9 (Fig. 5c). We observed dose-dependent increase in steady state Tfn-Alexa Fluor 633 accumulation with monensin (Fig. 5d). Next, we compared the effect of monensin and NHE9 overexpression on the kinetics of transferrin uptake

Figure 2 | Altering NHE9 expression in GBM BTICs affects tumour proliferation and invasion in vivo. (a-d) Two different MRI views for 276 (a,b) and 612 (c,d) GBM patients show the source of the BTIC used in our study. (e) Schematic of primary tumour extraction, subsequent culture in serum-free medium and intracranial injection into a mouse brain (coordinates: X1.5, Y-1.34, Z3.5 from Bregma). Blue dotted line in the illustrated brain section represents tumour area and green box represents cells migrating into the contralateral hemisphere. (f,g) Representative images of 4',6-diamidino-2phenylindole (DAPI)-stained coronal sections of mouse brains, after injection of GBM 612 BTIC transfected with (f) control shRNA or (g) NHE9 shRNA constructs. Yellow staining represents human GBM cells identified by monoclonal lgG1 antibody against human nuclei. (h-k) Effect of NHE9 on tumour size and migration. Orthotopic in vivo tumours formed by GBM 612 BTIC treated with NHE9 shRNA were significantly smaller (h) and less invasive (j) than their control counterpart. Tumours formed from GBM 276 BTIC were significantly larger (i) in cells overexpressing NHE9 and more invasive (k) than control cells. Bars represent mean \pm s.d.; ${ }^{\star \star} P<0.01,{ }^{\star \star \star} P<0.0001$, two-tailed Welch's $t$-test; from left to right, $n=27,28$ (h) and 19, 17 (i). See related Supplementary Fig. 3. 
a
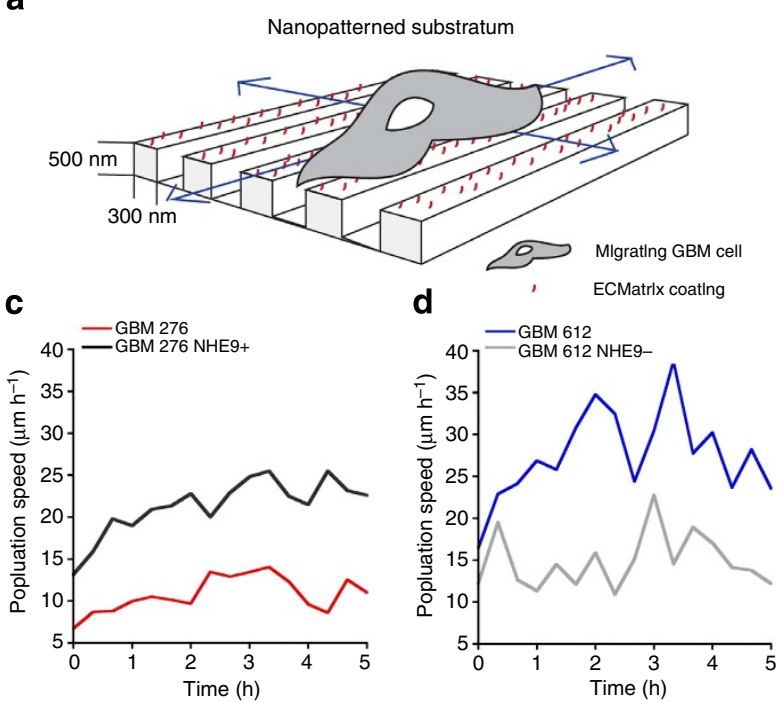

e

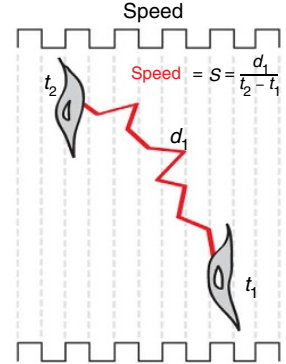

h

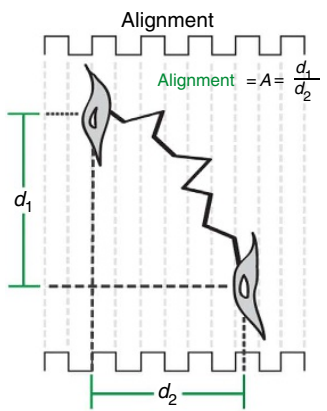

k

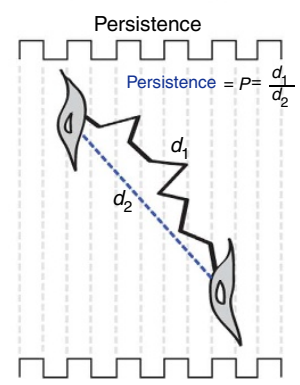

f

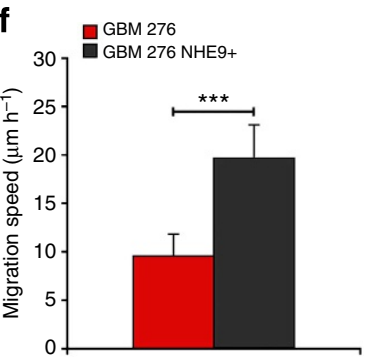

i

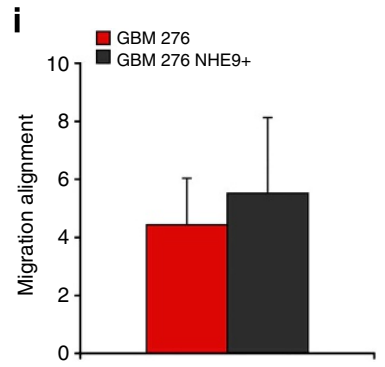

I

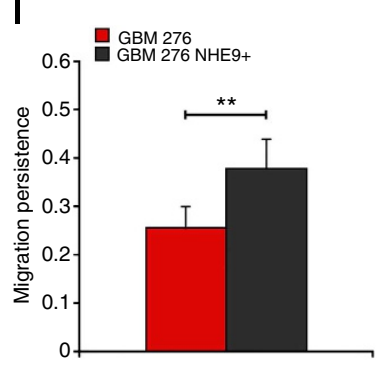

b

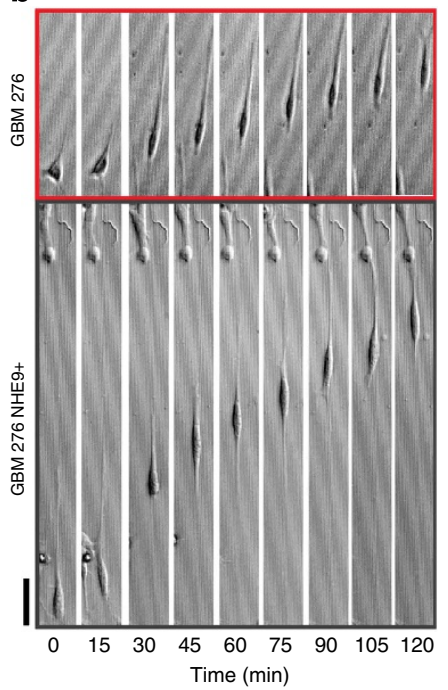

g GBM 612

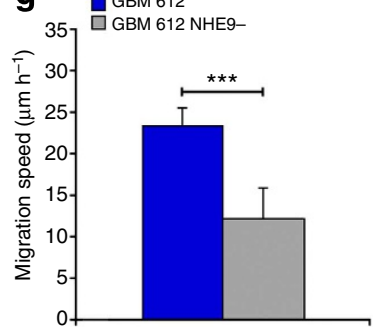

j

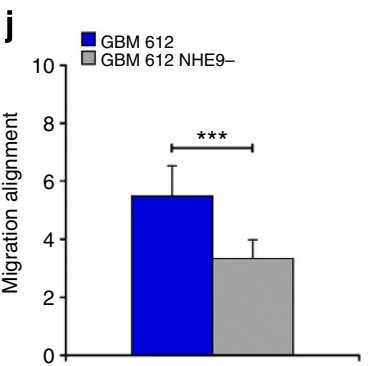

m

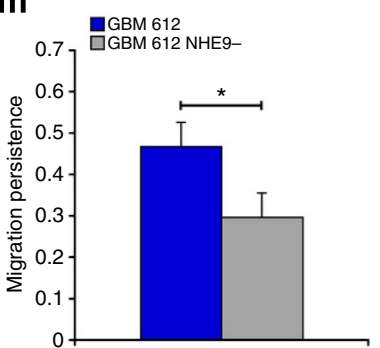

Figure 3 | GBM BTIC migration on a nanopatterned substrate is affected by NHE9. (a) Laminin-coated, longitudinal nanopatterned tracks with the designated dimensions were used for analysis of BTIC migration. (b) Representative time-lapse microscopic images collected every 15 min compare the migration of GBM 276 cells transfected with vector control and NHE9 over a period of $2 \mathrm{~h}$. Population speed, over a period of $5 \mathrm{~h}$, of GBM 276 (c) and GBM 612 (d) cells relative to NHE9 overexpression or knockdown as indicated. Schematic representations of migration speed (e), alignment (h) and persistence (k) of GBM BTICs are shown, along with the formulae used for quantification. GBM 276 BTICs transduced with lentiviral vectors overexpressing NHE9 migrate faster (f) and are more persistent (I) than control cells ( ${ }^{\star \star} P=0.0004,{ }^{\star \star \star} P=3.69 \times 10^{-11}$, two-tailed Welch's $t$-test, $n=75$ ). GBM 612 BTICs treated with NHE9 shRNA show a decreased migration speed $(\mathbf{g})$, alignment $(\mathbf{j})$ and persistence $(\mathbf{m})$ when compared with control virus-transduced cells $\left({ }^{\star \star \star} P<0.0001,{ }^{\star} P=0.036\right.$, two-tailed Welch's $t$-test, $\left.n=75\right)$. There was no significant difference in the alignment between control and NHE9overexpressing GBM 276 BTICs (i). Bars represent mean \pm s.d. Scale bar, $50 \mu \mathrm{m}$ (c). 

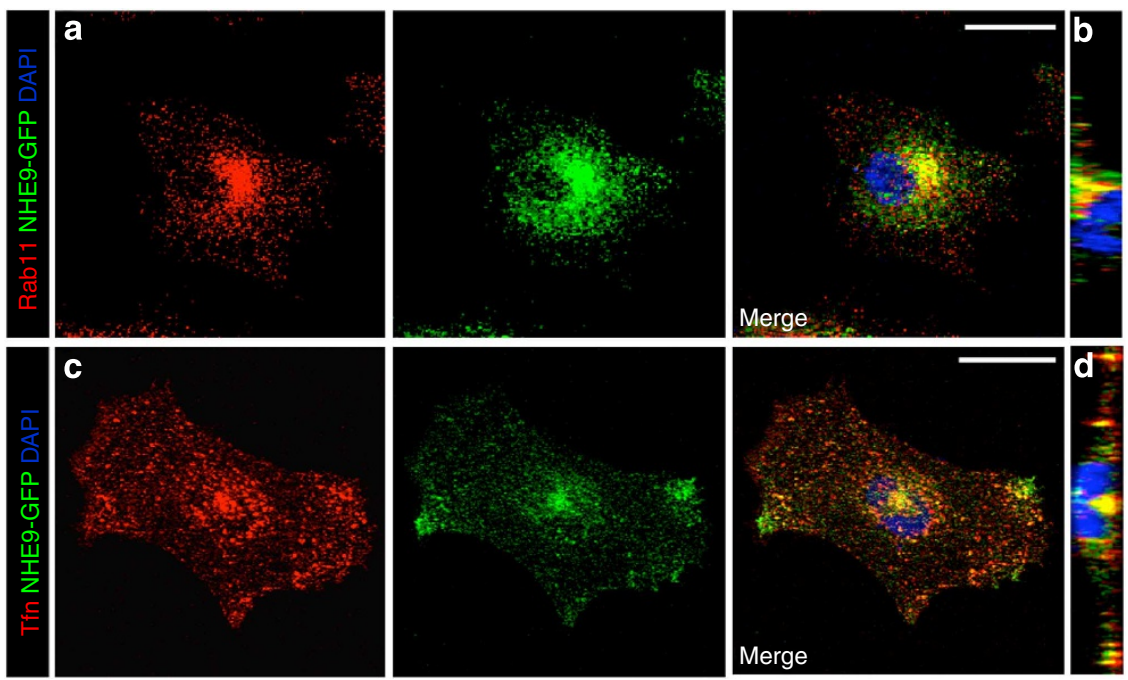

e

Steady-state transferrin uptake
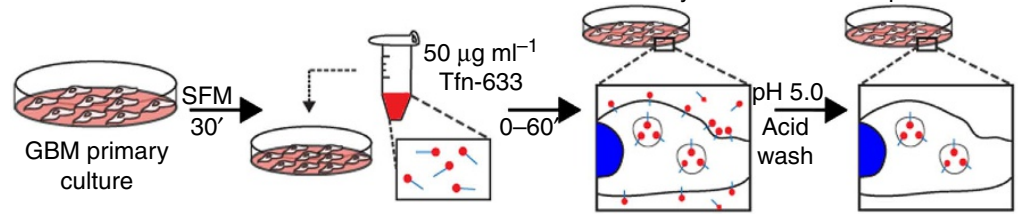

Flow

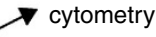

f

GBM 253

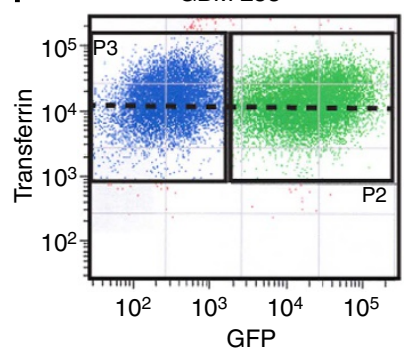

g

GBM 253 NHE9+
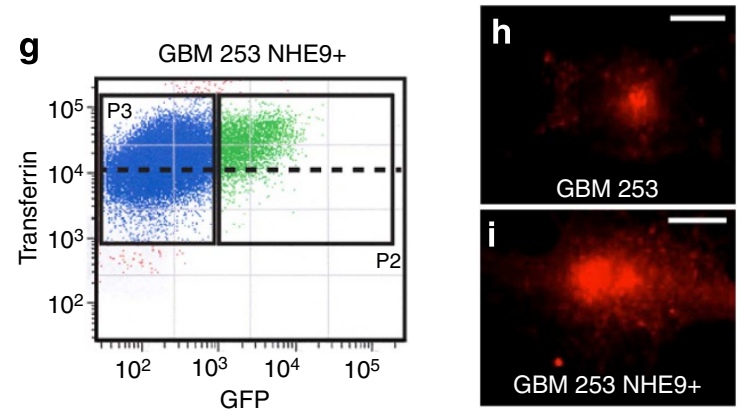

j

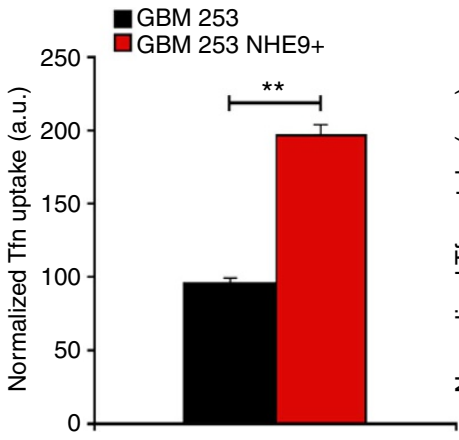

k

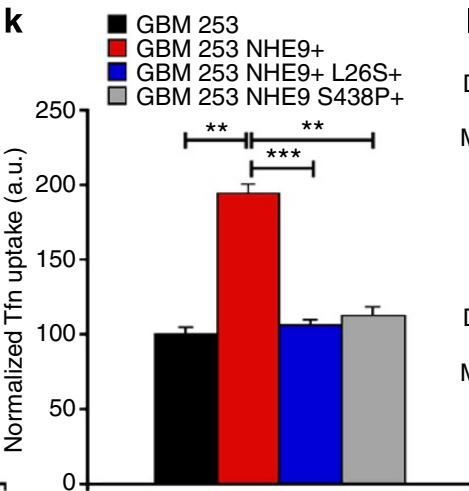

I

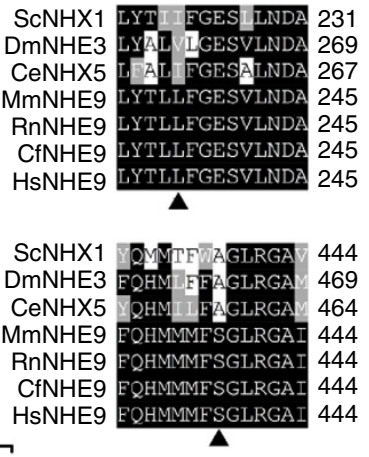

Figure 4 | NHE9 localizes to recycling endosomes and alters transferrin uptake. (a-d) Representative confocal microscopy images of GBM 253 cells expressing NHE9-GFP. Cells were fixed with 4\% PFA and Rab11, and observed by immunofluorescence. Subcellular co-localization of NHE9-GFP (green) with Rab11 (red) is seen in the merge (a) and orthogonal slice (b). Cells were incubated with transferrin tagged to Alexa Fluor-633 (Tfn-633) for 55 min and imaged (c) to show overlap with NHE9 in the perinuclear region and cell periphery, as seen in the orthogonal slice (d). (e) GBM 253 cells were transfected with GFP vector or NHE9-GFP and incubated with Tfn-633 for 55 min, to compare steady-state transferrin uptake. (f-g) GBM 253 cells were subjected to fluorescence-activated cell sorting (FACS) analysis to separate untransfected cells (blue) from GFP-expressing cells (green). Black dashed line indicates average fluorescence intensity ( $y$ axis) of GFP positive ( $x$ axis) population. There was an increase in average fluorescence of Tfn-633 in cells expressing NHE9-GFP relative to the GFP control, as seen by shift in the population over the black line. (h-i) Fluorescence micrograph of GBM 253 cells treated with Tfn-633 as in $\mathbf{f}$ and $\mathbf{g}$, respectively. Fluorescence intensity and exposure settings were the same in the two images. Following background subtraction, fluorescence signal was increased by 2.8 -fold in $\mathbf{i}$ relative to $\mathbf{h}$. (j) Quantitation of Tfn-633 fluorescence from FACS analysis in GBM 253 control cells and 253 cells overexpressing NHE9 ( ${ }^{\star} P=0.0005$, two-tailed Welch's $t$-test, $n=3$ with $>5,000$ cells). Note that the population in P2 may underestimate low expressors of NHE9-GFP in panel 5G. (k) Previously described loss-of-function mutations in NHE9 (L236S and S438P) failed to elevate Tfn-633 accumulation ${ }^{\star \star} P<0.01,{ }^{\star \star \star} P=2.56 \times 10^{-7}$, two-tailed Welch's $t$-test, $n=3$ ). (I) NHE9 and gene orthologue alignments showing residue conservation of loss-of-function mutations in various species. Bars represent mean \pm s.d. Scale bars, $20 \mu \mathrm{m}$. See related Supplementary Figs 4 and 5. 
and efflux. NHE9 overexpression increased the rate and steadystate levels of transferrin accumulation, whereas it had no effect on the rate of efflux (Fig. 5e-h). In contrast, the increase in transferrin accumulation observed with monensin treatment was largely due to a decrease in the rate of efflux (Fig. 5e-h). Thus, although both NHE9 and monensin alkalinized endosomal compartments to similar extent, they had distinct effects on transferrin uptake and efflux. It was possible that enhanced

a

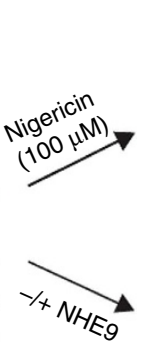
SFM, 30
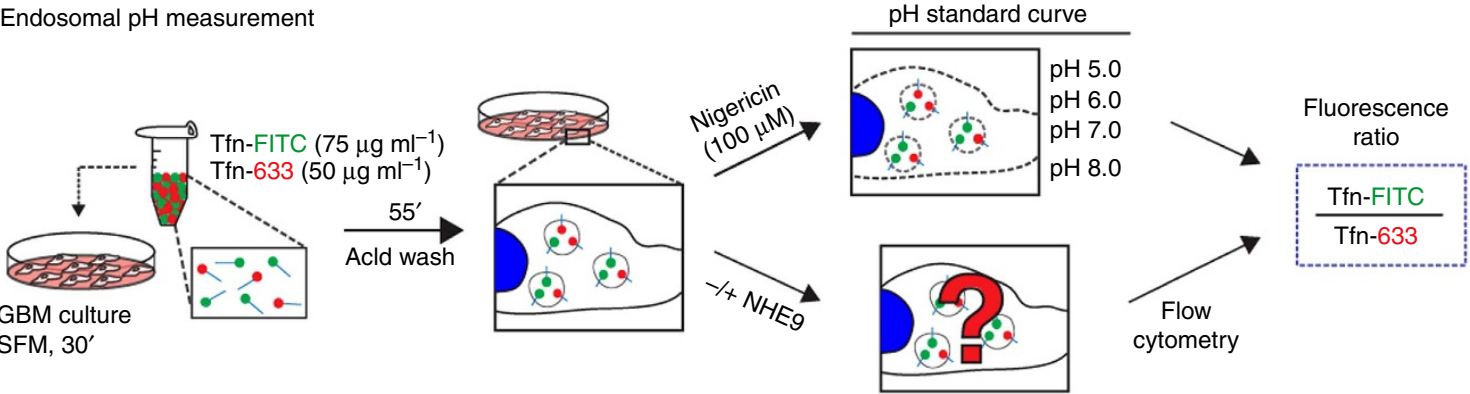

b

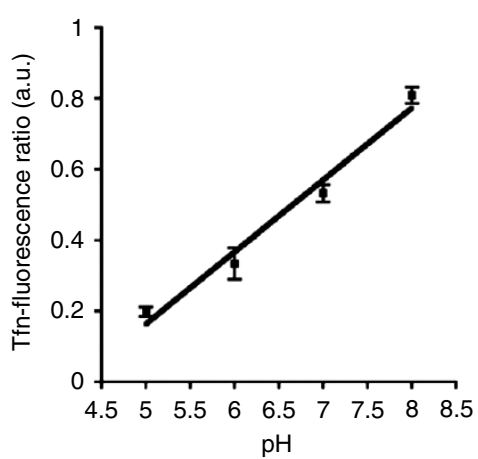

e $\quad 0$ min
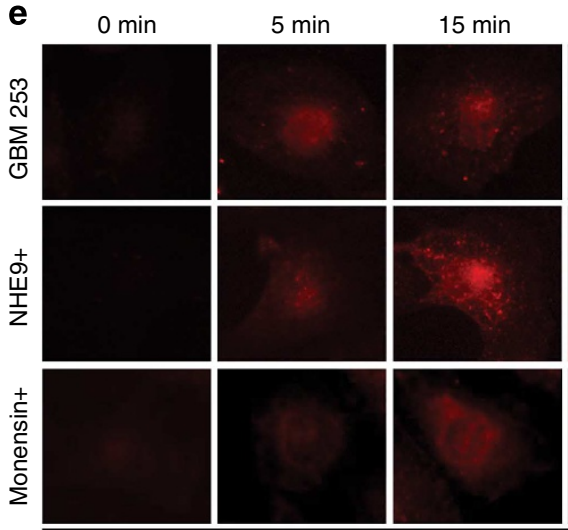

\section{C}
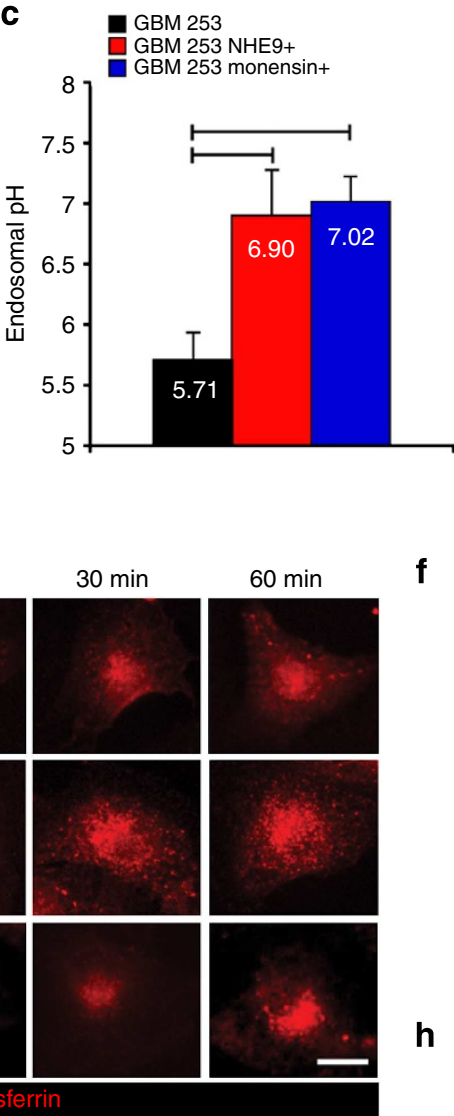

$90 \mathrm{~min}$
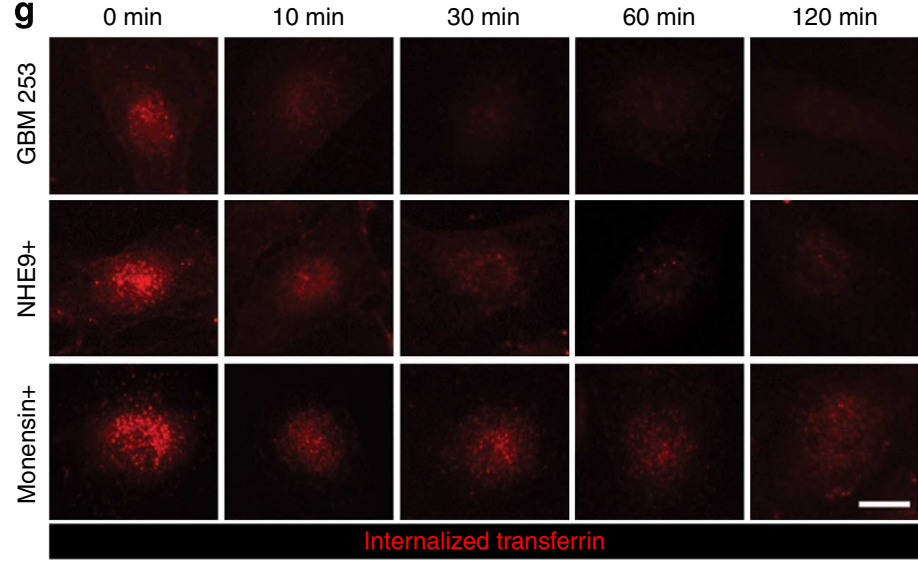

$120 \min$ d
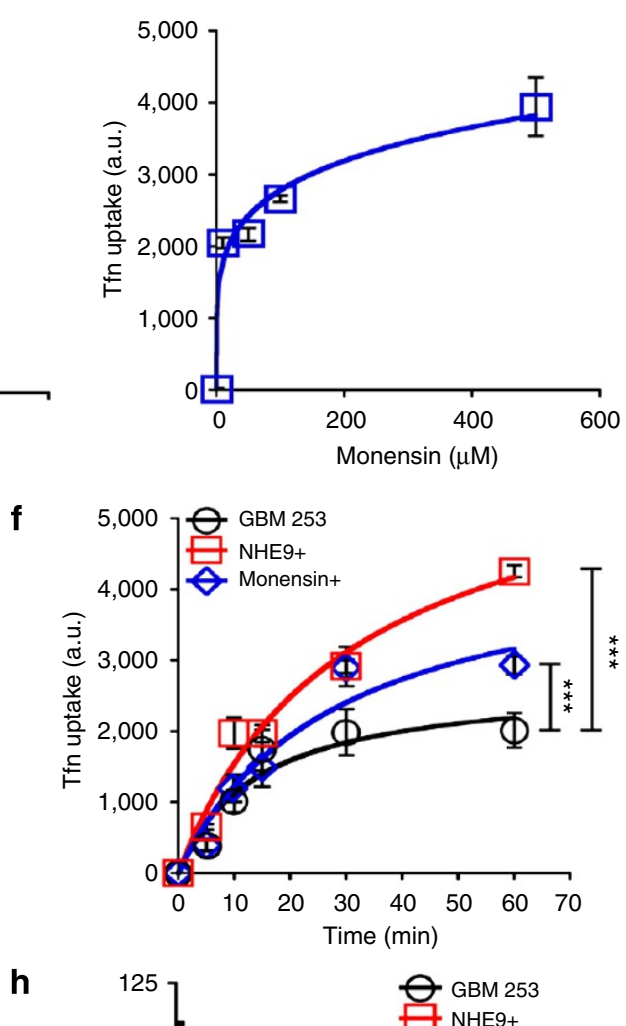

h
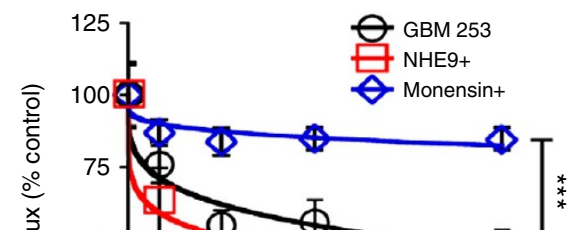

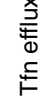
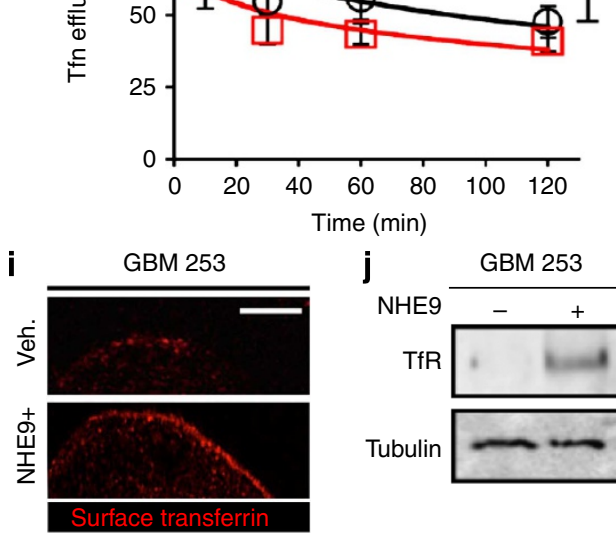

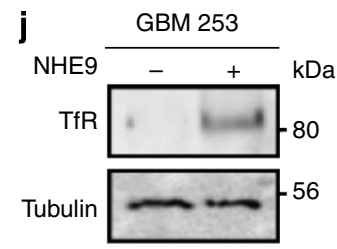


transferrin uptake resulting from ectopic expression of NHE9 was the result of elevated surface expression of the transferrin receptor on GBM 253 tumour cells. To test this hypothesis, we observed transferrin-Alexa Fluor 633 bound to GBM 253 cells on ice. Under these conditions, endocytosis was greatly reduced, allowing transferrin receptors present on the cell surface to be visualized. More surface-bound fluorescence was observed in GBM 253 cells transfected with NHE9 (Fig. 5i), consistent with elevated levels of transferrin receptor, which was confirmed by surface biotinylation (Fig. 5j). These findings suggest that NHE9 elevates surface levels of membrane receptors on GBM cells. In summary, we used a variety of experimental approaches, including flow cytometry, confocal microscopy, surface labelling and biotinylation in NHE9-overexpressing or monensin-treated cells, to demonstrate that endosomal $\mathrm{Na}^{+} / \mathrm{H}^{+}$exchange activity can strongly affect binding and uptake of transferrin at the cell membrane of GBM BTIC. These observations, if extended to other cell surface proteins and growth factor receptors, have the potential to impact oncogenic signalling.

NHE9 promotes EGFR signalling. A striking and signature feature of GBM is dysregulated EGFR activity, with gene amplification, mutation and alteration in receptor tyrosine kinase pathways driving tumour growth in over $80 \%$ of gliomas ${ }^{22}$. Although EGFR signalling pathways are compelling targets for therapeutic intervention, clinical outcomes for the first generation of anti-EGFR therapeutics have been disappointing in patients with GBM. This lack of efficacy of tyrosine kinase domain inhibitors has been attributed to redundancy in signalling pathways, tumour heterogeneity and mutation-specific differences in inhibitor sensitivity ${ }^{23}$. An independent and alternative strategy to inhibiting receptor tyrosine kinase phosphorylation is to downregulate cell surface availability of the receptor by exploiting negative feedback mechanisms known to control the duration and intensity of EGFR signalling. Based on our finding that NHE9 modulated transferrin receptor levels to regulate ligand uptake, we next investigated the effect of NHE9 on EGFR signalling in GBM BTIC. Growth rates of both GBM 276 and GBM 612 were responsive to EGF and were further increased or decreased, respectively, by NHE9 expression or knockdown (Supplementary Fig. 6A,B). Significantly, proliferation was lowest in the absence of EGF and NHE9 expression, suggesting that NHE9 may synergize with EGFR signalling to promote tumour cell growth (Supplementary Fig. 6A,B). EGFR transcript levels were the same in GBM 612 and GBM 276 (Supplementary Fig. 6C). Strikingly, however, plasma membrane expression of EGFR, as determined by surface biotinylation, was nearly threefold higher in the faster growing and more aggressive GBM 612 BTIC, relative to GBM 276 (Supplementary Fig. 6D). These findings suggested that differences in NHE9 expression between the two GBM cell lines were critical in determining surface expression of EGFR. Confirming this hypothesis, pair-wise experiments indicated that elevation of NHE9 expression in GBM 276 significantly increased plasma membrane persistence of EGFR relative to vector-transfected control (Fig. 6a,c). Conversely, transcriptional attenuation of NHE9 in GBM 612 cells significantly decreased surface expression of EGFR (Fig. 6b,d). Consistent with our findings, there was no correlation in gene expression between NHE9 and EGFR in the TCGA data set ${ }^{14}$ (Pearson correlation: $-0.157, P$-value: $0.141, n=91)$, and the subset of patients with mutations in EGFR (15 of 90 patients) was not associated with changes in survival or in NHE9 expression. Thus, we concluded that NHE9 functions posttranscriptionally to regulate EGFR signalling.

Stimulation with EGF triggers receptor endocytosis and lysosomal degradation, resulting in disappearance of $\sim 70 \%$ EGFR in GBM 276 cells and $\sim 36.4 \%$ in GBM 612 cells within $3 \mathrm{~h}$, in the absence of new protein synthesis (Fig. 6e,g, respectively). These differences in EGFR turnover could be directly linked to NHE9 levels. Thus, elevated expression of NHE9 in GBM 276 limited EGFR degradation to 15\%, whereas attenuation of NHE9 expression in GBM 612 tumour cells greatly enhanced EGFR turnover by $\sim 96.4 \%$ of initial levels, within $3 \mathrm{~h}$ (Fig. 6f,i and h,j, respectively). Next, we investigated the impact of altered NHE9 expression on EGFR signalling in GBM BTIC. NHE9 knockdown in GBM 612 decreases phosphorylation of EGFR (Fig. 6k,l) and key downstream signalling components that mediate tumour proliferation and invasion, including ERK, Cyclin D and AKT (Fig. 6m). Consistent with these findings, increased NHE9 expression in GBM 276 cells resulted in increased activation of the EGFR signalling pathway (Supplementary Fig. 6E).

Receptor sorting and degradation are dependent on acidic luminal $\mathrm{pH}$ in the endo-lysosomal system. We measured endosomal pH, as described for GBM 253 in Fig. 5, in GBM 276 and GBM 612. As predicted, $\mathrm{pH}_{\mathrm{e}}$ correlated with NHE9 expression in the three GBM cell lines. GBM 276, showing lowest NHE9 levels, also had lowest endosomal pH $(5.57 \pm 0.13)$, whereas GBM 612, which had the highest NHE9 levels, had the highest $\mathrm{pH}_{\mathrm{e}}(6.88 \pm 0.13)$. Furthermore, ectopic expression of NHE9 in GBM 276 significantly alkalinized $\mathrm{pH}_{\mathrm{e}}(6.61 \pm 0.21$; $P=0.023)$ and knockdown of NHE9 in GBM 612 resulted in corresponding acidification (to $6.63 \pm 0.095 ; P=0.049$ ). Together, these data indicate that dysregulation of EGFR signalling by

\section{Figure 5 | NHE9 regulates endosomal pH in GBM BTICs and controls transferrin receptor trafficking. (a) Endosomal pH of transferrin-positive} compartments was measured by treating GBM 253 cells with transferrin tagged to pH-sensitive fluorophore (Tfn-FITC) and the pH-insensitive Alexa-633 (Tfn-633). Control and NHE9-overexpressing cells, as well as cells used to calculate a pH calibration curve, were loaded with tagged Tfn for $1 \mathrm{~h}$ and a fluorescence ratio of internalized transferrin was quantified using flow cytometry. (b) Calibration of GBM 253 endosomal pH. Cells loaded with Tfn-FITC and Tfn-633 were exposed to nigericin $(100 \mu \mathrm{M})$ and assayed in pH-defined medium ( $\mathrm{pH}$ 5.0-8.0) to calculate their fluorescence ratio $\left(R^{2}=0.988\right)$. (c) $\mathrm{pH}$ in Tfn-positive endosomal compartments is alkalinized in GBM 253 cells expressing NHE9 or after treating with the ionophore monensin (500 $\mu \mathrm{M}$ for $1 \mathrm{~h}$ ) relative to vector control ( ${ }^{\star} P=0.032,{ }^{\star \star} P=0.002$, two-tailed Welch's $t$-test, $n=3$ ). (d) Dose dependence of Tfn-633 in response to monensin treatment ( $n=100$ cells per concentration). Fluorescence is presented in arbitrary units (a.u.). (e-f) Time course of Tfn-633 accumulation in GBM 253 cells transfected with vector control, NHE9 or treated with monensin $(500 \mu \mathrm{M})$, as indicated. Representative images are shown (e) and mean fluorescence \pm s.d. was plotted (f). Cells expressing NHE9 and control cells treated with monensin show an increased rate and total accumulation ( ${ }^{\star \star \star} P<0.0001$, two-tailed Welch's $t$-test, $n=3$ with $>75$ cells). ( $\left.\mathbf{g}-\mathbf{h}\right)$ Time course of Tfn- 633 efflux was monitored by loss of fluorescence, after loading cells for $55 \mathrm{~min}$, as seen in representative images $(\mathbf{g})$ and plot of mean fluorescence \pm s.d. (h). Monensin treatment drastically reduces Tfn-633 efflux, whereas overexpression of NHE9 did not significantly change Tfn efflux ( ${ }^{* \star} P<0.0001$, two-tailed Welch's $t$-test, $n=3$ with $>75$ cells). Plasma membrane levels of transferrin receptor (TfR) in GBM 253 cells were monitored by a surface-binding assay performed on ice that only allows Tfn-633 to bind surface receptors (i) and by western blot analysis of surface biotinylation (j). GBM 253 cells expressing NHE9 showed increased surface expression of TfR ( $n=3$ ). Bars represent mean \pm s.d. Scale bars, $20 \mu \mathrm{m}$. 
a

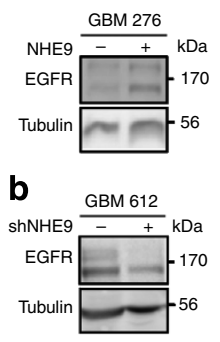

C

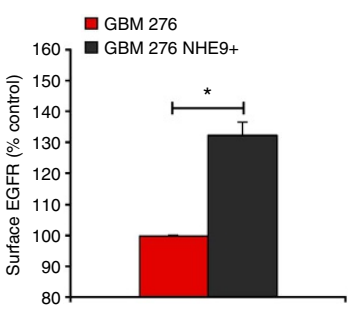

d

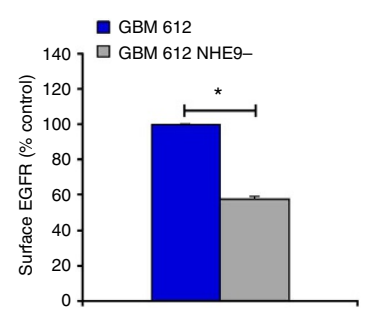

e

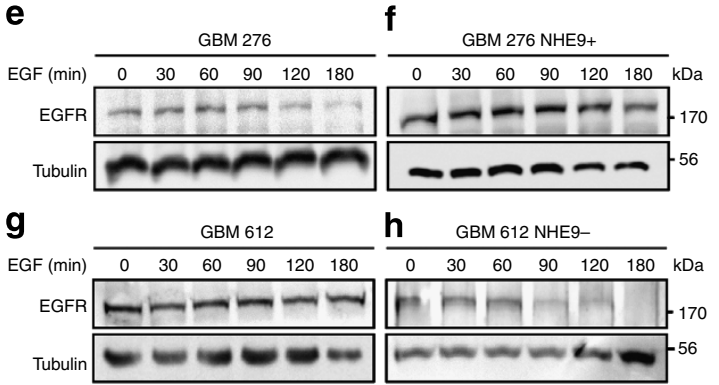

i

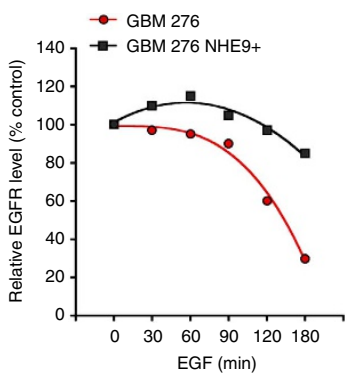

j

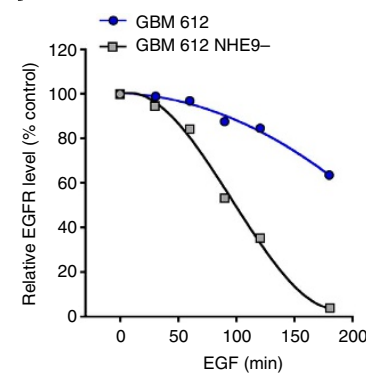

k

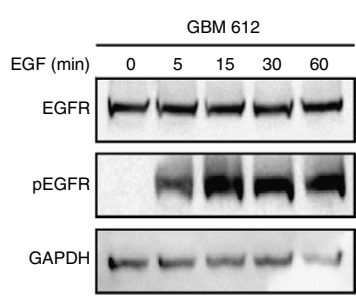

I

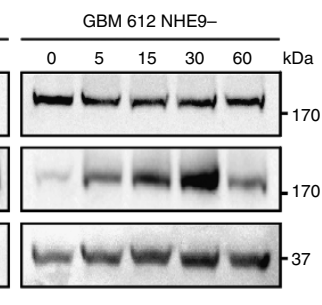

m

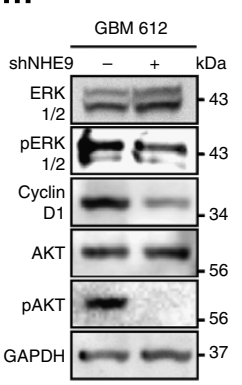

Figure 6 | NHE9 drives EGFR membrane persistence and downstream oncogenic signalling in GBM BTICs. (a-d) Plasma membrane levels of EGFR, determined by surface biotinylation, are elevated in GBM 276 cells expressing NHE9-GFP (a,c) and decreased in GBM 612 cells treated with NHE9-shRNA (b,d). Representative blots are shown for GBM 276 with NHE9 overexpression (a) and GBM 612 virally transfected with NHE9 shRNA (b). Graphs represent average band intensity from densitometric scans of immunoblottings. EGFR levels were normalized to Tubulin and are shown relative to vector-transformed control ( ${ }^{\star} P<0.05$, two-tailed Welch's $t$-test, $n=3$ ). (e-h) NHE9 regulates EGFR turnover in GBM BTICs. Cells were growth factor starved and pre-incubated with cycloheximide $(\mathrm{CHX})$ for $2 \mathrm{~h}$, followed by the addition of EGF $\left(20 \mathrm{ng} \mathrm{ml}^{-1}\right)$. At time intervals indicated, cell lysates were collected and analysed by western blotting. Overexpressing NHE9 in GBM 276 (f) hampered EGFR degradation induced by EGF stimulation compared with 276 control cells (e). GBM 612 cells with attenuated NHE9 expression (h) showed the opposite affect, with increased EGFR degradation compared with 612 vector controls (g). Representative blots for both GBM 276 and GBM 612 are shown $(n=3)$. EGFR bands in the CHX assay (e-h) were normalized to their respective Tubulin levels and graphed as a percentage of GBM 276 (i) or GBM 612 (j) controls without the addition of EGF. Knockdown of NHE9 expression in GBM 612 cells downregulates the activation of EGFR (I) compared with vector control (k). Cells were growth factor starved for $2 \mathrm{~h}$ and then treated with EGF for the indicated times before lysates were collected for immunoblotting $(n=3)$. Downstream signalling pathways of GBM 612 ( $\mathbf{m}$ ) treated with EGF (30 min) were also altered in cells transfected with NHE9 shRNA. Cell lysates were immunoblotted with the indicated antibodies. Bars represent mean \pm s.d. See related Supplementary Fig. 6.

NHE9-mediated endosomal $\mathrm{pH}$ regulation could be one of the critical mechanisms leading to tumour progression.

Therapeutic potential of NHE9 inhibition. To explore the potential of NHE9 as a therapeutic target in a subset of GBMs, we first tested the effect of NHE inhibitors on GBM BTIC proliferation. The amiloride analogue 5-( $N$-ethyl- $N$-isopropyl) amiloride (EIPA) is a general NHE inhibitor ${ }^{24}$. The IC $_{50}$ for EIPA was $100 \mu \mathrm{g} \mathrm{ml}^{-1}$ for GBM 276 tumour cells and increased by $20 \%$ following overexpression of NHE9 (Fig. 7a), consistent with NHE9 being a target for EIPA. We also observed dose-dependent reversal of the NHE9-mediated increase in transferrin uptake in GBM 253 cells by EIPA (Supplementary Fig. 7A). Lower doses of EIPA, sufficient to inhibit NHE1, failed to inhibit growth of GBM 276 or reverse NHE9-mediated increase in cell proliferation (Supplementary Fig. 7B). Overexpression of NHE9 greatly decreased the efficacy of Erlotinib, a known tyrosine kinase inhibitor of EGFR. Addition of EIPA $\left(100 \mu \mathrm{g} \mathrm{ml}^{-1}\right)$ effectively reversed the drug resistance conferred by NHE9, decreasing $\mathrm{IC}_{50}$ for erlotinib from 52 to $31 \mu \mathrm{g} \mathrm{ml}^{-1}$ relative to that of the control GBM 276 cells, $17 \mu \mathrm{g} \mathrm{ml}^{-1}$ (Fig. 7b). Similarly, treatment with EIPA improved inhibition of GBM 612 cells by Erlotinib (Supplementary Fig. 7C). We show that EIPA reduces EGFR expression in GBM 612 BTIC on addition of EGF (Fig. 7c,d), consistent with the increased efficacy of Erlotinib following EIPA treatment. U0126, a known mitogen-activated protein kinase (ERK1/2) inhibitor, also decreased the cell proliferation in GBM
276 cells overexpressing NHE9 (Supplementary Fig. 7D), suggesting that the proliferation effects of NHE9 in these GBM cells were partly mediated by mitogen-activated protein kinase signalling, a major effector pathway in the EGFR network.

Renewable tumoursphere formation (Fig. 7e) is associated with tumorigenic capacity and rapid tumour progression, and is a significant predictor of clinical outcome ${ }^{25}$. Knockdown of NHE9 in GBM 612 cells reduced the number of tumourspheres by $\sim 1$.6-fold compared with control cells (Fig. 7f,h,i). The diameter of the tumourspheres was also reduced by $\sim 3.3$-fold (Fig. $7 g-i$ ). Treating GBM 612 cells with $100 \mu \mathrm{g} \mathrm{ml}^{-1}$ of EIPA drastically reduced both the number and diameter of tumourspheres by $\sim 7$-fold (Fig. $7 \mathrm{f}-\mathrm{h}, \mathrm{j}$ ). Although the EIPA effects could include non-selective inhibition of other NHE isoforms besides NHE9, our results support the therapeutic potential of NHE9 as a drug target for a subset of GBM expressing elevated NHE9 levels. In such cases, bimodal therapy with known EGFR inhibitors could be an effective therapy.

\section{Discussion}

A common pathophysiological hallmark of tumours is a perturbation in $\mathrm{pH}$ dynamics ${ }^{26}$. Oncogene-mediated elevation of cytoplasmic $\mathrm{pH}$ by constitutive activation of $\mathrm{NHE} 1$ and ensuing extracellular acidification have long been recognized as an early step in neoplastic transformation driven by ras and $\mathrm{c}-\mathrm{mos}^{27,28}$. In contrast, the role of endosomal $\mathrm{pH}$ modulation in oncogenesis has not been studied, although there is emerging 
a

b
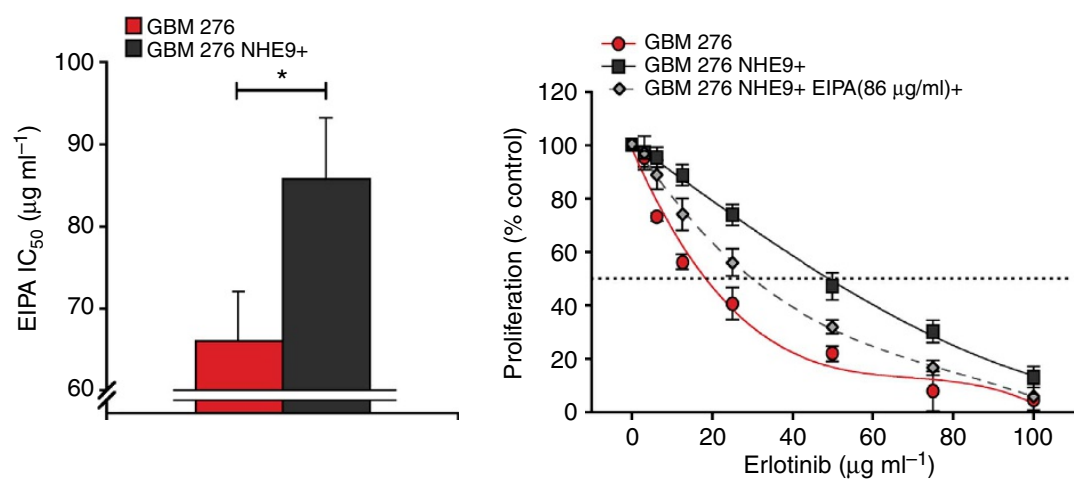

f Tumoursphere assay

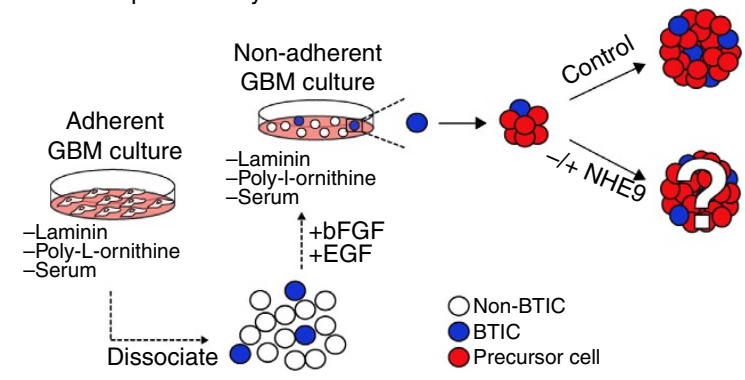

h

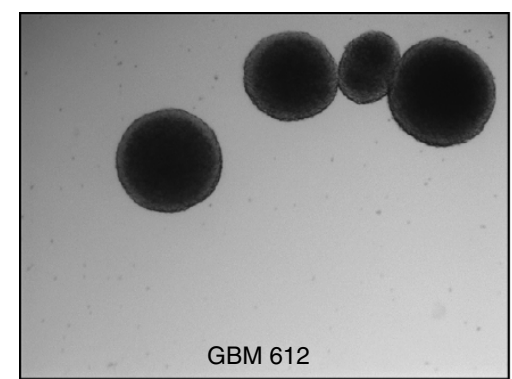

i

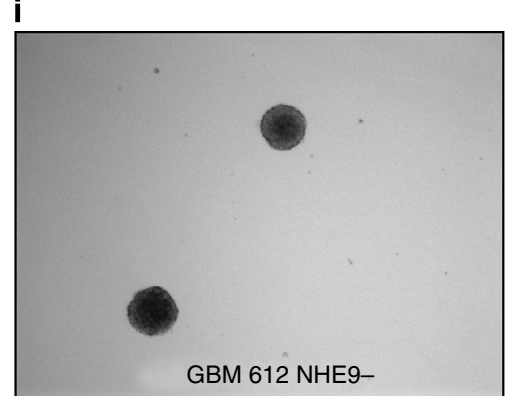

C

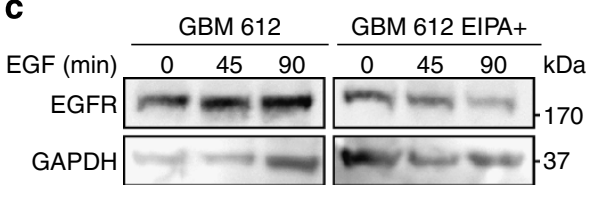

d

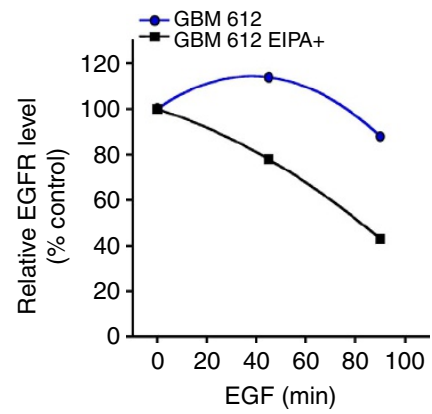

9

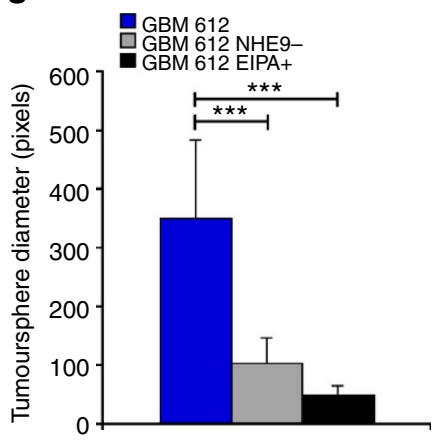

j

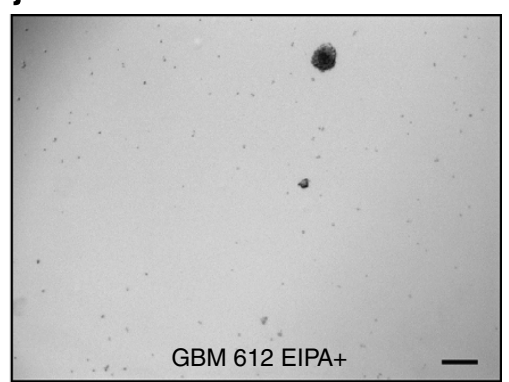

Figure 7 | NHE inhibitor EIPA mitigates resistance to Erlotinib, EGFR persistence and tumoursphere formation in GBM BTICs. (a) GBM 276 cells were treated for $24 \mathrm{~h}$ in the presence of different concentrations of EIPA and cell viability was measured with a colorimetric MTS assay. The graph shows $\mathrm{IC}_{50}$ for EIPA is elevated in cells overexpressing NHE9 relative to vector control ( ${ }^{\star} P<0.05$, two-tailed Welch's $t$-test, $n=3$ ). (b) GBM 276 control cells, NHE9-overexpressing cells and NHE9-overexpressing cells treated for $24 \mathrm{~h}$ with $85.83 \mu \mathrm{g} \mathrm{ml}^{-1}$ (EIPA IC 50 of 276 NHE9+ cells) were treated with different concentrations of EGFR inhibitor Erlotinib and cell viability was measured by MTS assay $(n=3)$. (c-d) GBM 612 cells were growth factor starved, pre-incubated with cycloheximide (CHX) for $2 \mathrm{~h}$ and treated with EGF $\left(20 \mathrm{ng} \mathrm{ml}^{-1}\right)$ for the times indicated before cell lysates were collected and analysed by western blotting. Six hundred and twelve cells treated with EIPA $\left(85.83 \mu \mathrm{g} \mathrm{ml}^{-1}\right)$ showed an increase in EGF-stimulated EGFR degradation compared with vector control $(\mathbf{c})$ and representative blots are shown $(n=3)$. EGFR bands in $\mathbf{c}$ were normalized to their GAPDH control and graphed as a percentage of controls (d) without the addition of EGF. (e) Adherent GBM BTICs not cultured in the presence of EGF were dissociated and transferred to non-adherent conditions (without laminin or poly-L-ornithine coating). Cells were cultured in StemPro NSC SFM media with EGF $\left(20 \mathrm{ng} \mathrm{ml}^{-1}\right.$ ) for 21 days before tumoursphere formation was analysed. (f-g) Image J software was used to determine the number (f) and diameter ( $(\mathbf{g})$ of tumourspheres from GBM 612, GBM 612 cells transfected with NHE9 shRNA and GBM 612 cells treated with EIPA $\left(85.83 \mu \mathrm{g} \mathrm{ml}{ }^{-1} ;{ }^{\star} P<0.05,{ }^{\star \star} P<0.01,{ }^{\star \star \star} P<0.0001\right.$, two-tailed Welch's $t$-test, $n=3)$. $(\mathbf{h}-\mathbf{j})$ Representative phase-contrast images of the tumourspheres are shown. Scale bar, $50 \mu \mathrm{m}$. Bars represent mean $\pm \mathrm{s}$.d. See related Supplementary Fig. 7.

evidence implicating organellar $\mathrm{Na}^{+} / \mathrm{H}^{+}$exchangers in various cancers. In hypermutated colorectal tumours, SLC9A9, the gene encoding NHE9, was found to be among the most frequently mutated targets along with $A C V R 2 A, A P C, T G F B R 2, M S H 3$, MSH6 and TCF7L2 (ref. 29). Consistent with this finding, a genome-wide scan for colorectal cancer susceptibility identified linkage to chromosome 3q, with SLC9A9 being one of the candidate genes ${ }^{30}$. Furthermore, a genome-wide case-control study of ESCC including 300 ESCC cases and 300 matched controls discovered significant associations of single-nucleotide polymorphisms in SLC9A9 with ESCC risk $^{31}$. The link between NHE9 and ESCC is strengthened by a recent study demonstrating 
a

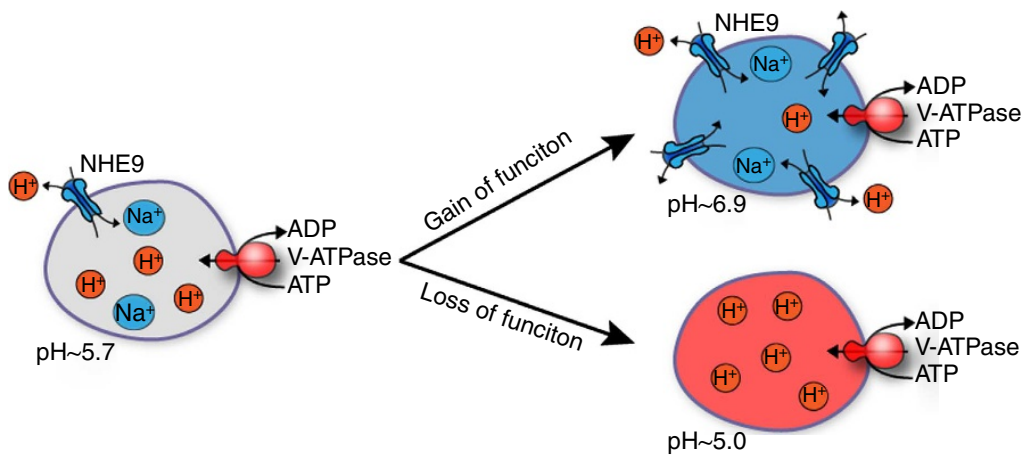

b
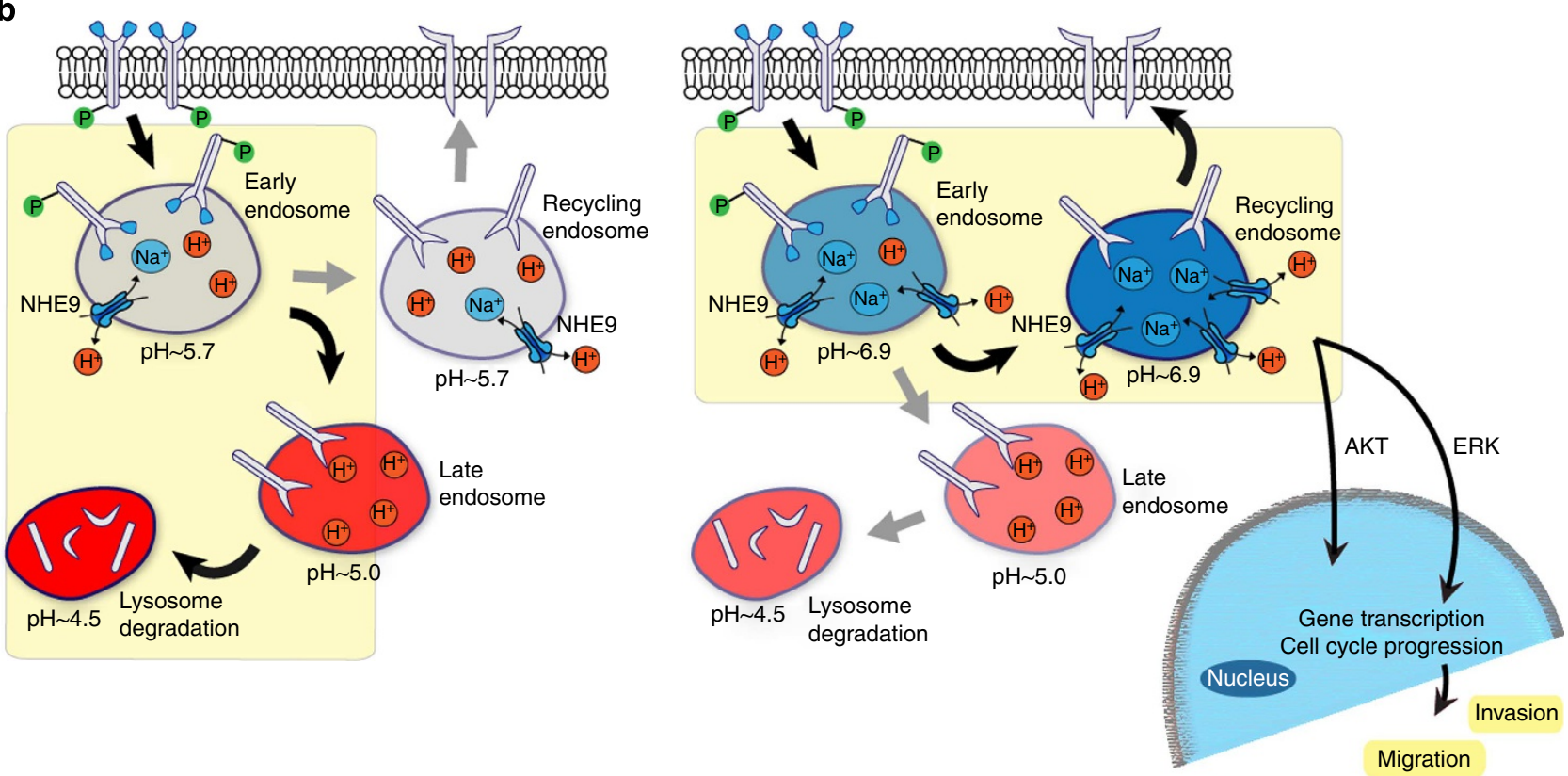

Cell proliferation

Figure 8 | Model for endosomal pH regulation by NHE9 and its affect on the downstream EGFR network. (a) Endosomal acidification is regulated by the V-ATPase, which pumps protons into the endosome lumen, and NHE9, which allows for proton movement out of the endosome via exchange with $\mathrm{Na}^{+}$or $\mathrm{K}^{+}$. The endosome is alkalinized when there is proton leakage as a result of increased NHE9 expression or activity, and inversely acidified when there is a loss of NHE9 function. (b) Endosomes are trafficked through a series of pH-defined compartments that can lead either to (i) recycling back to the plasma membrane, or (ii) degradation within the lysosome. Luminal pH could regulate the activity of acid hydrolases and/or the sorting of endosomes along distinct pathways. A major fate of endocytosed EGFR under high ligand conditions is delivery to lysosomes for degradation. The model proposes that endosomal alkalization resulting from increased NHE9 activity diverts EGFR trafficking away from lysosomes and thus increases its membrane persistence. This results in activation of oncogenic downstream signals that lead to increased cell migration and tumorigenesis.

that patients with ESCC with NHE9 downregulation ( $>2$-fold) showed complete response to neoadjuvant chemo-radiotherapy ${ }^{17}$. Despite these clinical and genetic links, the molecular role of NHE9 in carcinogenesis was previously unknown. There has been one report showing that ectopic expression of NHE7, a closely related isoform of NHE9 that localizes to the trans-Golgi network, increased tumorigenicity in a breast cancer cell line ${ }^{32}$. Although the underlying mechanisms were not investigated, the study showed that whereas tumour invasion was enhanced by both NHE1 and NHE7, only NHE7 could promote anchorageindependent growth and cell adhesion.

There is new evidence from model organisms as well as mammalian cells that luminal $\mathrm{pH}$ in the secretory-endosomal pathway is set by a balance of proton pump and leak, and is critical for normal vesicle trafficking (Fig. 8a) ${ }^{33-35}$. Although the importance of the $\mathrm{H}^{+}$pumping V-ATPase is firmly established, we are only beginning to appreciate the complementary role of eNHE as $\mathrm{a} \mathrm{H}^{+}$leak pathway for the precise tuning of luminal
pH. Pathophysiological elevation or loss of NHE9 activity is associated with glioblastoma (this study) or autism, ${ }^{6,7}$, as a consequence of excessive luminal alkalization and acidification, respectively (Fig. 8a). Early endosomes transit through a series of increasingly acidic compartments, culminating with delivery and degradation of cargo at the lysosome (Fig. 8b, left panel). Luminal $\mathrm{pH}$ affects the fate of cargo, both by modulating acid-activated hydrolases and by directing vesicle traffic through one of the several routes. Thus, premature activation of vacuolar acid hydrolases in the yeast mutant lacking the eNHE orthologue, NHX1, results in degradation of chaperone protein VPS10 in the prevacuolar compartment and missorting of cargo to the extracellular medium ${ }^{36}$. Similarly, enhanced turnover of EGFR resulting from silencing or inhibition of NHE9 may be due to acidification of the endolysosomal system and activation of acid hydrolases. On the other hand, receptors may escape degradation by recycling back to the plasma membrane instead of being sorted to the late endosome/lysosome (Fig. 8b; right panel). We show 
that elevated levels of plasma membrane receptors seen in NHE9-overexpressing GBM cells greatly enhance Alexa Fluorlabelled transferrin uptake by TfnR-mediated endocytosis and downstream signalling from EGFR.

The EGFR signalling network is a powerful driver of glioblastoma ${ }^{37}$. Ligand-bound EGFR not only activates intracellular tyrosine kinases directly but synergistically transactivates other cell surface receptors to ignite signalling cascades that control cell proliferation, invasion and apoptosis. Yet, despite the importance of EGFR signalling, multiple clinical trials using EGFR kinase inhibitors have had disappointing clinical outcomes due to their lack of tumour cytotoxicity. Although kinase inhibition of EGFR results in decreased cell proliferation, cell death appears to require the loss of EGFR protein, consistent with vastly different in vivo phenotypes of EGFR knockout and mutants with greatly attenuated kinase activity $^{38,39}$. The resistance of GBM to EGFR kinase inhibitors has been attributed to a host of confounding factors, including activation of compensatory pathways, constitutively active mutations and kinase-independent prosurvival roles of EGFR. For example, EGFR protects cancer cells from autophagic death by stabilizing the active glucose transporter SGLT1, independent of its kinase function ${ }^{40}$. There is growing realization that targeting EGFR for degradation could be a more effective strategy than inhibiting kinase activity. Recently, it was shown that depletion or inhibition of a dual specificity tyrosine phosphorylation-regulated kinase, DYRK1A, targets EGFR for lysosomal degradation, severely compromising self-renewal ability of GBM BTIC ${ }^{41}$. Similarly, the tumour suppressor Mig-6 protein recruits internalized EGFR to the late endosome for eventual lysosomal degradation, by association with the SNARE protein STX8 (ref. 42). Another example is that of LRIG, a transmembrane protein that controls CBL recruitment and EGFR downregulation, found to be attenuated in many glioblastomas ${ }^{43,44}$. Taken together, several independent studies have borne out that EGFR membrane persistence is key to oncogenic activity.

Resveratrol, a potent SIRT1 activator, has anti-tumour property ${ }^{45}$. Treatment of GBM BTIC with resveratrol showed significant downregulation of NHE9 that is associated with reduced tumorigenicity and enhanced radiosensitivity ${ }^{46}$. In this context, it is worth noting that NHE9 is a known SIRT1 (sirtuin 1)-associated gene and is repressed with SIRT1 activation ${ }^{47}$. We show that increased NHE9 expression in a subset of GBM patients decreases survival and promotes membrane persistence of multiple glial receptors and transport proteins, including EGFR, TfR (this study) and glutamate transporter ${ }^{6}$. As multiple receptor tyrosine kinases are coactivated in $\mathrm{GBM}^{48}$, we propose that endosomal NHE9 exerts pan-specific control over plasma membrane receptor density. Previous studies using three-dimensional multi-scale brain tumour models suggest that a high EGFR density triggers the phenotypic switch between proliferation and migration early in the development of aggressive gliomas ${ }^{49,50}$. Further, the simulations indicate that spatio-temporal expansion of glioblastoma depends largely on posttranslational events, and that malignancy cannot be determined by monitoring gene expression data alone. Our findings validate these predictions: although EGFR transcript levels were the same, the more aggressive GBM 612 tumour had much higher plasma membrane levels of EGFR protein relative to the slower growing and less invasive GBM 276 cells. We show that high levels of NHE9 correlate with increased receptor density by ensuring membrane persistence of EGFR irrespective of transcript levels, and that manipulating NHE9 expression or activity has profound effect on brain tumour stem cell renewal, proliferation and invasion. We note that membrane transport proteins are eminently 'druggable' and have been successfully targeted by blockbuster drugs (Prozac, Prilosec and Lasix, to name a few). Pharmaceutical companies have already developed cardioprotective drugs against NHE1, another isoform in the NHE family. Our findings highlight an urgent need to screen for high affinity, selective NHE9 inhibitors that could be used in conjunction with receptor tyrosine kinase inhibitors to enhance receptor clearance as an effective treatment for glioblastoma.

\section{Methods}

GBM cell cultures. All glioblastoma tumour samples were obtained with written informed consent from patients through the Department of Neurosurgery, Johns Hopkins Hospital (Baltimore, MD), following Institutional Review Board guidelines and as previously described ${ }^{2,51,52}$. Patient tumour grade and type was determined by a neuropathologist (Supplementary Table 1). Culture conditions were in a $5 \% \mathrm{CO}_{2}$ incubator at $37^{\circ} \mathrm{C}$ as follows: GBM 253 primary glioblastoma cells were subcultured in DMEM/F12 (1:1) Nutrient Mixture, 10\% fetal bovine serum (Invitrogen) and 1\% HyClone Antibiotic Antimycotic Solution (Thermo Scientific). GBM 276 and GBM 612 glioblastoma stem cells were cultured in one of two ways: (1) as serum-free cultures on laminin and poly-L-ornithine-coated plates $^{53}$ using StemPro NSC SFM (Invitrogen) without EGF, or (2) as tumoursphere cultures with EGF $\left(20 \mathrm{ng} \mathrm{ml}^{-1}\right)$. EGF was only added to adherent cell cultures during experimental procedures.

Plasmids. Full-length mNHE9-EGFP was cloned into FuGW lentiviral vector as previously described ${ }^{6}$. Quik-Change-Site-Directed Mutagenesis Kit was used to make all point mutations (Stratagene). HsNHE9 short hairpin RNA (shRNA) sequence $\left(5^{\prime}\right.$-CCGGCCCTCCATTAAGGAGAGTTTTTCAAGAGAAAACTCTC CTTAATGGAGGTTTTTC- $3^{\prime}$ ) and control shRNA sequence (Sigma-Aldrich) $\left(5^{\prime}\right.$-CAACAAGATGAAGAGCACCAA- $\left.3^{\prime}\right)$ were cloned into pLKO.1 lentiviral vector with a puromycin-selectable marker.

Construction of lentiviruses and cell transduction. pCMV-dR8.9 and pMD2.G packaging plasmids were co-transfected with pLKO.1-NHE9 shRNA in HEK293 cells for $48 \mathrm{~h}$. FuGW mNHE9-EGFP was co-transfected 1:1 with 2nd Generation Packaging Mix (ABM) into HEK293 cells according to manufacturer's instructions. Virus was collected 48 and $72 \mathrm{~h}$ after transfection and Lenti-X Concentrator (Clontech) was added to concentrate lentiviral stocks. Glioblastoma cells infected with shRNA constructs were incubated with virus for $48 \mathrm{~h}$ and selected with puromycin $\left(0.75 \mathrm{\mu g} \mathrm{ml}^{-1}\right)$. GBM cells infected with mNHE9-EGFP virus were assayed $72 \mathrm{~h}$ after infection.

Quantitative PCR analysis. mRNA was isolated from cell cultures using the RNeasy Mini kit (Qiagen), following the manufacturer's instructions, and optional RNA treatment with DNase I (Roche) was done. Complementary DNA was synthesized using the High-Capacity RNA-to-cDNA Kit (Applied Biosystems, 4387406), following the manufacturer's instructions. Quantitative real-time PCR analysis was performed using the 7500 Real-Time PCR System (Applied Biosystems) using Taqman Fast universal PCR Master Mix (Applied Biosystems, 4352042). Taqman gene expression assay probes used were Hs00234723_m1 (SLC9A6/NHE6), Hs00543518_m1 (SLC9A9/NHE9), Hs00184593_m1 (ATP6AP1/V-ATPase subunit), Hs01076078_m1 (EGFR), Hs01009250_m1 (PROM1/CD133), Hs99999901_s1 (18S RNA) and Hs02758991_g1 (GAPDH). Each experiment was performed with following quantitative PCR cycling conditions: $2 \mathrm{~min}$ at $50^{\circ} \mathrm{C}, 10 \mathrm{~min}$ at $95^{\circ} \mathrm{C}$, then 40 cycles of $15 \mathrm{~s}$ at $95^{\circ} \mathrm{C}$ and $1 \mathrm{~min}$ at $60^{\circ} \mathrm{C}$. Ct values were used for all manipulations and were first normalized to endogenous control levels by calculating the $\Delta \mathrm{Ct}$ for each sample. Values were then calculated relative to control, to generate a $\Delta \Delta \mathrm{Ct}$ value. Fold change was calculated using the equation: expression fold change $=2^{-\Delta \Delta \mathrm{Ct}}$. Three technical replicates of three independent (biological) replicates were run to account for assay variance.

Cell proliferation assay. Cell growth was monitored using the Celltiter 96 Aqueous One Solution Cell Proliferation Assay (MTS (3-(4,5-dimethylthiazol2-yl)-5-(3-carboxymethoxyphenyl)-2-(4-sulfophenyl)-2H-tetrazolium), Promega), which is based on metabolic activity as a proxy for cell number ${ }^{54}$. Briefly, $0.5 \times 10^{4}$ cells were seeded, in triplicate, into a 96 -well plate. At each time point, $20 \mu \mathrm{l}$ of Celltiter 96 Aqueous One Solution reagent was added to each well and incubated for $2 \mathrm{~h}$ at $37^{\circ} \mathrm{C}$. The absorbance was measured at $490 \mathrm{nM}$ using a 96 -well plate reader and normalized to $\mathrm{D} 1$.

Intracranial GBM injections and immunohistochemistry. All animal protocols were approved by the Johns Hopkins Animal Care and Use Committee. In vivo tumorigenesis and invasion of cells expressing either NHE9-GFP or NHE9 shRNA were assayed in 4- to 6-week-old nude/athymic, male mice ( $\mathrm{NCr}$-nu/nu, $\mathrm{NCI}$ ) using a previously described brain tumour model ${ }^{55}$. Briefly, a total of 150,000 cells were injected into the right hemisphere of the mouse brains (coordinates X1.5, 
Y1.34, Z3.5 relative to bregma). Each experimental group had eight animals each. Animals dying before the termination of the experiment were excluded from the analysis. Mice were killed 8 weeks after injection, and fixed and stained as previously described ${ }^{2}$. Briefly, mice were subjected to a transcardiac perfusion using $4 \%$ paraformaldehyde as fixative. Dissected brain were postfixed overnight at $4{ }^{\circ} \mathrm{C}$ in the same fixative, embedded in OCT compound (Tissue-Tek), frozen at $-80^{\circ} \mathrm{C}$ and sectioned. Then, cryosections were stained with an antibody against human nuclei (mouse monoclonal IgG1, 1:250, Millipore) and were imaged to calculate tumour size and invasiveness by morphometric analysis using Image J (NIH). The slides were randomized, coded in a blinded manner and counted to prevent observer bias.

Nanopatterned cell migration and quantitative analysis. GBM cell migration was assessed and analysed using a previously described directional migration assay $^{2}$. Briefly, nanogrooved substrates made of transparent polyurethane acrylate were constructed using ultraviolet-assisted capillary lithography. The nanopatterned surface was coated with laminin and cell migration was quantified using time-lapse microscopy. Observation was done using a Cascade 512B II CCD camera equipped to an Olympus IX81 inverted microscope in a chamber controlled for temperature and environmental factors. Phase-contrast cell images were recorded automatically under $\times 10$ objective for $15 \mathrm{~h}$ at 15 -min intervals using Slidebook 4.1 (Intelligent Imaging Innovations).

A previously designed custom MATLAB script was used to allow manual tracking of cells frame-by-frame. Beginning from the centre of the cell body, average individual cell speed was calculated from a cell trajectory tracing the centroid position between intervallic images recorded within the 15-h duration. Cell persistence was calculated by fractioning the shortest distance between the start and end point of a given cell's centroid position over the total distance travelled. Cell alignment to the nanopatterned substratum was calculated by fractioning the distance moved parallel over the distance moved perpendicularly to the ridges. All analysis were stopped in the case of an abnormal event (cell death, division and so on) and not used in the final average calculations. All averages were calculated using a minimum of 75 cell trajectories.

Immunofluorescence. Cultured GBM cells on coverslips were pre-extracted with PHEM buffer (60 mM PIPES, $25 \mathrm{mM}$ HEPES, $10 \mathrm{mM}$ EGTA and $2 \mathrm{mM} \mathrm{MgCl}_{2}$, $\mathrm{pH}$ 6.8) containing $0.025 \%$ saponin for $2 \mathrm{~min}$, then washed twice for $2 \mathrm{~min}$ with PHEM buffer containing $0.025 \%$ saponin and $8 \%$ sucrose. The cells were fixed with a solution of $4 \%$ paraformaldehyde and $8 \%$ sucrose in PBS for $30 \mathrm{~min}$ at room temperature, and blocked with a solution of $1 \%$ BSA and $0.025 \%$ saponin in PBS for $1 \mathrm{~h}$. Primary antibodies were diluted 1:100 in 1\% BSA and incubated with cells $1 \mathrm{~h}$ or overnight. Alexa Fluor 568 goat anti-rabbit IgG and Alexa Fluor 568 goat anti-mouse IgG (both Invitrogen) were used at 1:1,000 dilution for $30 \mathrm{~min}$. Cells were mounted onto slides using Dako Fluorescent Mounting Medium and were imaged using a Zeiss LSM510-Meta confocal microscope.

pH measurement and transferrin uptake. Cells were rinsed and incubated in serum-free medium for $30 \mathrm{~min}$, to remove any residual transferrin. Later on, cells were exposed to $100 \mu \mathrm{g} \mathrm{ml}^{-1}$ transferrin conjugated to Alexa Fluor 633 (Invitrogen) for imaging or flow cytometry, or to $100 \mu \mathrm{g} \mathrm{ml}^{-1}$ transferrin conjugated to $\mathrm{pH}$-sensitive FITC $\left(75 \mu \mathrm{g} \mathrm{ml}^{-1}\right)$ and $\mathrm{pH}$ non-sensitive Alexa Fluor 633 $\left(25 \mu \mathrm{g} \mathrm{ml}^{-1}\right)$ (Invitrogen) for $\mathrm{pH}$ measurement at $37^{\circ} \mathrm{C}$ for $55 \mathrm{~min}$. Transferrin uptake was stopped by chilling the cells on ice. External transferrin was removed by washing with ice-cold serum-free DMEM and PBS, whereas bound transferrin was removed by an acid wash in PBS at pH 5.0 followed by a wash with PBS at pH 7.0. Surface-bound transferrin ( $<5 \%$ to total) was determined with a parallel sample incubated on ice and was used for background subtraction. The fluorescence intensity of internalized transferrin was measured for at least 5,000 cells by flow cytometry using the FACSAria (BD Biosciences) instrument and the average intensity of the cell population was recorded ${ }^{6}$.

For transferrin endocytosis and exocytosis measurements, cells on coverslips were rinsed and incubated in serum-free medium for $30 \mathrm{~min}$ and then exposed to $100 \mathrm{\mu g} \mathrm{ml}^{-1}$ transferrin conjugated with Alexa Fluor 633 for varying amounts of time. Washes were done accordingly (as described) and cells were mounted onto slides using Dako Fluorescent Mounting Medium. Fluorescence microscopy images were acquired with a Photometrics CoolSNAP FX digital camera and processed using Scanalytics IPLab software and Adobe Photoshop CS2 software. Internal fluorescence was quantified using ImageJ software, $n=100$, for each time point for each experiment. Exocytosis was measured as a loss of fluorescence at various time points similarly.

The surface-binding assay was carried out similarly. Cells on coverslips were rinsed and incubated in serum-free medium for $30 \mathrm{~min}$ and then exposed to $100 \mu \mathrm{g} \mathrm{ml}^{-1}$ transferrin conjugated with Alexa Fluor 633 in 1\% BSA solution on ice for $2 \mathrm{~min}$, followed by subsequent washes with PBS at pH 7.0. Cells were mounted on slides and imaged on a Zeiss LSM510-Meta confocal microscope.

Cytoplasmic $\mathrm{pH}$ was measured using flow cytometry as previously described ${ }^{56}$. Briefly, cells were incubated with $1 \mu \mathrm{M}$ BCECF-AM (Life Technologies) for $30 \mathrm{~min}$ at $37^{\circ} \mathrm{C}$, and $\mathrm{pH}$ was determined by excitation at $488 \mathrm{~nm}$ and emissions filtered through $530( \pm 15) \mathrm{nm}(\mathrm{pH}$-sensitive green fluorescence) and $616( \pm 12) \mathrm{nm}$
( $\mathrm{pH}$ non-sensitive red fluorescence) filters. A $\mathrm{pH}$ calibration curve was generated by preloading cells with $1 \mu \mathrm{M}$ BCECF-AM for $30 \mathrm{~min}$ followed by incubation in a 'high $\mathrm{K}^{+}$' HEPES buffer $\left(25 \mathrm{mM}\right.$ HEPES, $145 \mathrm{mM} \mathrm{KCl}, 0.8 \mathrm{mM} \mathrm{MgCl}_{2}, 1.8 \mathrm{mM}$ $\mathrm{CaCl}_{2}, 5.5 \mathrm{mM}$ glucose), whose $\mathrm{pH}$ was adjusted to different values $(6.6,7.0,7.4$ and 7.8$)$ and used to prepare a four-point calibration curve in the presence of $\mathrm{K}^{+} / \mathrm{H}^{+}$ionophore nigericin $(10 \mu \mathrm{M})$.

Protein turnover and western blotting. Cells were lysed with Nonidet P-40 ( $1 \%$, Sigma) that included protease inhibitor cocktail (Roche) and then centrifuged for $15 \mathrm{~min}$ at 14,000 r.p.m. at $4{ }^{\circ} \mathrm{C}$. Cell protein lysates (50-100 $\left.\mu \mathrm{g}\right)$ were dissolved in loading buffer (62.5 mM Tris- $\mathrm{HCl} \mathrm{pH} \mathrm{6.8,} \mathrm{10 \%} \mathrm{glycerol,} \mathrm{2 \%} \mathrm{SDS,} 0.01 \%$ Bromophenol Blue, $100 \mathrm{mM}$ dithiothreitol) and separated by SDS-PAGE. Antibodies used for western blotting were as follows: Tf Receptor (Abcam ab38171, 1:250); EGFR (Millipore 06-847, 1:250); p-EGFR, Tyr1068 (Cell Signaling 2234, 1:1,000); ERK1/2 (Sigma M 5670, 1:2,000); pERK1/2, Thr183 (Sigma M 7802, 1:500); Cyclin D1 (Sigma C 7464, 1:200); AKT (Cell Signaling 9272, 1:1,000); and pAKT, Ser 473 (Cell Signaling 4056, 1:1,000). Loading controls used were Tubulin (Sigma T 9026, 1:1,000) and GAPDH (Sigma G 9295, 1:20,000).

Cells used for degradation assays were plated at $2.5 \times 10^{5}$ cells per well in a six-well tissue culture dish and allowed to adhere overnight. The following day, cells were growth factor starved for $2 \mathrm{~h}$ in the presence of $50 \mu \mathrm{M}$ cycloheximide (Sigma). These conditions were maintained for the duration of the experiment. Following, $20 \mathrm{ng} \mathrm{ml}^{-1} \mathrm{EGF}$ (PeproTech) was added to the media for the indicated amounts of time. Cells were then chilled on ice, washed with PBS and protein lysates were collected for immunoblotting. Blots were cropped to show relevant bands.

Cell surface biotinylation. For surface biotinylation, cell surface proteins were labelled with biotin as previously described ${ }^{57}$. Briefly, cells were washed three times with ice-cold PBS and incubated with $1 \mathrm{mg} \mathrm{ml}^{-1}$ Sulpho-NHS-LC biotin in PBS at $4^{\circ} \mathrm{C}$ for $20 \mathrm{~min}$. Excess NHS groups were quenched using $100 \mathrm{mM}$ glycine followed by three washes with PBS. Protein supernatants were mixed with $120 \mu \mathrm{l}$ of immobilized Neutravidin beads and incubated at $4{ }^{\circ} \mathrm{C}$ overnight, with gentle rotation. Beads collected by centrifugation were washed three times with lysis buffer and surface proteins labelled with biotin were separated by SDS-PAGE and analysed by immunoblotting. All blots were cropped to show relevant bands.

Oncomine and CBioPortal data. Primary source for the GBM expression data on NHE9 obtained from Oncomine was from Lee et al..$^{15}$ Survival data obtained on the 91 'complete' sequenced tumours through the cBioPortal ${ }^{58}$ were from the Glioblastoma project TCGA data set ${ }^{13}$.

\section{References}

1. Chaichana, K. L. et al. Factors involved in maintaining prolonged functional independence following supratentorial glioblastoma resection. Clinical article. J. Neurosurg. 114, 604-612 (2011).

2. Garzon-Muvdi, T. et al. Regulation of brain tumor dispersal by NKCC1 through a novel role in focal adhesion regulation. PLoS Biol. 10, e1001320 (2012).

3. Agudelo-Garcia, P. A. et al. Glioma cell migration on three-dimensional nanofiber scaffolds is regulated by substrate topography and abolished by inhibition of STAT3 signaling. Neoplasia 13, 831-840 (2011).

4. Stupp, R. et al. Radiotherapy plus concomitant and adjuvant temozolomide for glioblastoma. N. Engl. J. Med. 352, 987-996 (2005).

5. Watkins, S. \& Sontheimer, H. Unique biology of gliomas: challenges and opportunities. Trends Neurosci. 35, 546-556 (2012).

6. Kondapalli, K. C. et al. Functional evaluation of autism-associated mutations in NHE9. Nat. Commun. 4, 2510 (2013).

7. Kondapalli, K. C., Prasad, H. \& Rao, R. An inside job: how endosomal $\mathrm{Na}+/ \mathrm{H}+$ exchangers link to autism and neurological disease. Front. Cell. Neurosci. 8, 172 (2014).

8. Morrow, E. M. et al. Identifying autism loci and genes by tracing recent shared ancestry. Science 321, 218-223 (2008).

9. Di Domenico, F. et al. Expression of human papilloma virus type 16 E5 protein in amelanotic melanoma cells regulates endo-cellular $\mathrm{pH}$ and restores tyrosinase activity. J. Exper. Clin. Cancer Res. 28, 4 (2009).

10. Suprynowicz, F. A. et al. The human papillomavirus type 16 E5 oncoprotein inhibits epidermal growth factor trafficking independently of endosome acidification. J. Virol. 84, 10619-10629 (2010).

11. Chakravarti, A., Dicker, A. \& Mehta, M. The contribution of epidermal growth factor receptor (EGFR) signaling pathway to radioresistance in human gliomas: a review of preclinical and correlative clinical data. Int. J. Radiat. Oncol. Biol. Phys. 58, 927-931 (2004).

12. Rhodes, D. R. et al. ONCOMINE: a cancer microarray database and integrated data-mining platform. Neoplasia 6, 1-6 (2004).

13. Network, T. C. Corrigendum: Comprehensive genomic characterization defines human glioblastoma genes and core pathways. Nature 494, 506 (2013). 
14. Cancer Genome Atlas Research Network Comprehensive genomic characterization defines human glioblastoma genes and core pathways. Nature 455, 1061-1068 (2008).

15. Lee, J. et al. Tumor stem cells derived from glioblastomas cultured in bFGF and EGF more closely mirror the phenotype and genotype of primary tumors than do serum-cultured cell lines. Cancer Cell 9, 391-403 (2006).

16. Verhaak, R. G. et al. Integrated genomic analysis identifies clinically relevant subtypes of glioblastoma characterized by abnormalities in PDGFRA, IDH1, EGFR, and NF1. Cancer Cell 17, 98-110 (2010).

17. Wen, J. et al. Gene expression analysis of pretreatment biopsies predicts the pathological response of esophageal squamous cell carcinomas to neochemoradiotherapy. Ann. Oncol. 25, 1769-1774 (2014).

18. Yim, E. K. et al. Nanopattern-induced changes in morphology and motility of smooth muscle cells. Biomaterials 26, 5405-5413 (2005).

19. Giese, A. \& Westphal, M. Glioma invasion in the central nervous system. Neurosurgery 39, 235-250; discussion 250-232 (1996).

20. Kim, D. H. et al. Mechanosensitivity of fibroblast cell shape and movement to anisotropic substratum topography gradients. Biomaterials 30, 5433-5444 (2009).

21. Xinhan, L. et al. $\mathrm{Na}+/ \mathrm{H}+$ exchanger isoform 6 (NHE6/SLC9A6) is involved in clathrin-dependent endocytosis of transferrin. Am. J. Physiol. Cell Physiol. 301, C1431-C1444 (2011).

22. Brennan, C. W. et al. The somatic genomic landscape of glioblastoma. Cell 155, 462-477 (2013).

23. Barkovich, K. J. et al. Kinetics of inhibitor cycling underlie therapeutic disparities between EGFR-driven lung and brain cancers. Cancer Discov. 2, 450-457 (2012).

24. Orlowski, J. \& Grinstein, S. Diversity of the mammalian sodium/proton exchanger SLC9 gene family. Pflugers Arch. 447, 549-565 (2004).

25. Laks, D. R. et al. Neurosphere formation is an independent predictor of clinical outcome in malignant glioma. Stem Cells 27, 980-987 (2009).

26. Webb, B. A., Chimenti, M., Jacobson, M. P. \& Barber, D. L. Dysregulated pH: a perfect storm for cancer progression. Nat. Rev. Cancer 11, 671-677 (2011).

27. Amith, S. R. \& Fliegel, L. Regulation of the $\mathrm{Na}+/ \mathrm{H}+$ exchanger (NHE1) in breast cancer metastasis. Cancer Res. 73, 1259-1264 (2013).

28. Reshkin, S. J., Greco, M. R. \& Cardone, R. A. Role of pHi, and proton transporters in oncogene-driven neoplastic transformation. Phil. Trans. R. Soc. Lond. B Biol. Sci. 369, 20130100 (2014).

29. Cancer Genome Atlas, N.. Comprehensive molecular characterization of human colon and rectal cancer. Nature 487, 330-337 (2012).

30. Picelli, S. et al. Genome-wide linkage scan for colorectal cancer susceptibility genes supports linkage to chromosome 3q. BMC Cancer 8, 87 (2008).

31. Ng, D. et al. Replication of a genome-wide case-control study of esophageal squamous cell carcinoma. Int. J. Cancer 123, 1610-1615 (2008).

32. Onishi, I. et al. Organellar $(\mathrm{Na}+, \mathrm{K}+) / \mathrm{H}+$ exchanger NHE7 regulates cell adhesion, invasion and anchorage-independent growth of breast cancer MDA-MB-231 cells. Oncol. Rep. 27, 311-317 (2012).

33. Brett, C. L., Donowitz, M. \& Rao, R. Evolutionary origins of eukaryotic sodium/ proton exchangers. Am. J. Physiol. Cell. Physiol. 288, C223-C239 (2005).

34. Brett, C. L., Wei, Y., Donowitz, M. \& Rao, R. Human $\mathrm{Na}(+) / \mathrm{H}(+)$ exchanger isoform 6 is found in recycling endosomes of cells, not in mitochondria. Am. J. Physiol. Cell. Physiol. 282, C1031-C1041 (2002).

35. Nakamura, N., Tanaka, S., Teko, Y., Mitsui, K. \& Kanazawa, H. Four $\mathrm{Na}+/ \mathrm{H}+$ exchanger isoforms are distributed to Golgi and post-Golgi compartments and are involved in organelle $\mathrm{pH}$ regulation. J. Biol. Chem. 280, 1561-1572 (2005).

36. Bowers, K., Levi, B. P., Patel, F. I. \& Stevens, T. H. The sodium/proton exchanger Nhxlp is required for endosomal protein trafficking in the yeast Saccharomyces cerevisiae. Mol. Biol. Cell 11, 4277-4294 (2000).

37. Kalman, B., Szep, E., Garzuly, F. \& Post, D. E. Epidermal growth factor receptor as a therapeutic target in glioblastoma. Neuromol. Med. 15, 420-434 (2013).

38. Miettinen, P. J. et al. Epithelial immaturity and multiorgan failure in mice lacking epidermal growth factor receptor. Nature 376, 337-341 (1995).

39. Luetteke, N. C. et al. The mouse waved-2 phenotype results from a point mutation in the EGF receptor tyrosine kinase. Genes Dev. 8, 399-413 (1994).

40. Weihua, Z. et al. Survival of cancer cells is maintained by EGFR independent of its kinase activity. Cancer Cell 13, 385-393 (2008).

41. Pozo, N. et al. Inhibition of DYRK1A destabilizes EGFR and reduces EGFR-dependent glioblastoma growth. J. Clin. Invest. 123, 2475-2487 (2013).

42. Ying, H. et al. Mig-6 controls EGFR trafficking and suppresses gliomagenesis. Proc. Natl Acad. Sci. USA 107, 6912-6917 (2010).
43. Mao, F. et al. A role for LRIG1 in the regulation of malignant glioma aggressiveness. Int. J. Oncol. 42, 1081-1087 (2013).

44. Ye, F. et al. Upregulation of LRIG1 suppresses malignant glioma cell growth by attenuating EGFR activity. J. Neurooncol. 94, 183-194 (2009).

45. Knutson, M. D. \& Leeuwenburgh, C. Resveratrol and novel potent activators of SIRT1: effects on aging and age-related diseases. Nutr. Rev. 66, 591-596 (2008).

46. Yang, Y. P. et al. Resveratrol suppresses tumorigenicity and enhances radiosensitivity in primary glioblastoma tumor initiating cells by inhibiting the STAT3 axis. J. Cell. Physiol. 227, 976-993 (2012).

47. Oberdoerffer, P. et al. SIRT1 redistribution on chromatin promotes genomic stability but alters gene expression during aging. Cell 135, 907-918 (2008).

48. Stommel, J. M. et al. Coactivation of receptor tyrosine kinases affects the response of tumor cells to targeted therapies. Science 318, 287-290 (2007).

49. Zhang, L., Strouthos, C. G., Wang, Z. \& Deisboeck, T. S. Simulating brain tumor heterogeneity with a multiscale agent-based model: linking molecular signatures, phenotypes and expansion rate. Math. Comput. Model. 49, 307-319 (2009).

50. Athale, C. A. \& Deisboeck, T. S. The effects of EGF-receptor density on multiscale tumor growth patterns. J. Theor. Biol. 238, 771-779 (2006).

51. Li, Y. et al. c-Met signaling induces a reprogramming network and supports the glioblastoma stem-like phenotype. Proc. Natl Acad. Sci. USA 108, 9951-9956 (2011).

52. Tzeng, S. Y. et al. Non-viral gene delivery nanoparticles based on poly(betaamino esters) for treatment of glioblastoma. Biomaterials 32, 5402-5410 (2011).

53. Pollard, S. M. et al. Glioma stem cell lines expanded in adherent culture have tumor-specific phenotypes and are suitable for chemical and genetic screens. Cell Stem Cell 4, 568-580 (2009).

54. Riss, T. L. et al. in Assay Guidance Manual (eds Gal-Edd, N., Sittampalam, G. S., Arkin, M., Auld, D., Austin, C., Bejcek, B., Glicksman, M., Inglese, J., Lemmon, V., Li, Z., McGee, J., McManus, O., Minor, L., Napper, A., Riss, T., Trask, O. J. \& Weidner, J.) (Eli Lilly \& Company and the National Center for Advancing Translational Sciences, 2013).

55. Guerrero-Cazarez, H., Attenello, F., Noiman, L. \& Quiñones-Hinojosa, A. Stem Cells in Gliomas (Elsevier, 2011).

56. Franck, P. et al. Measurement of intracellular $\mathrm{pH}$ in cultured cells by flow cytometry with BCECF-AM. J. Biotechnol. 46, 187-195 (1996).

57. Ohgaki, R., Fukura, N., Matsushita, M., Mitsui, K. \& Kanazawa, H. Cell surface levels of organellar $\mathrm{Na}+/ \mathrm{H}+$ exchanger isoform 6 are regulated by interaction with RACK1. J. Biol. Chem. 283, 4417-4429 (2008).

58. Gao, J. et al. Integrative analysis of complex cancer genomics and clinical profiles using the cBioPortal. Sci. Signal. 6, pl1 (2013).

\section{Acknowledgements}

This work was supported by grants from the National Institutes of Health NS070024 (A.Q.H.), and DK054214 and GM62142 (R.R.). K.C.K. was supported by an American Heart Association postdoctoral fellowship (11POST7380034); J.P. received support from the Johns Hopkins PREP program; H.P. was a Fulbright Fellow supported by the International Fulbright Science and Technology Award; and A.H. was a Porter fellow of the American Physiological Society.

\section{Author contributions}

K.C.K. and J.P. conceived and performed all in vitro experiments with GBM 276 and 612; V.C.-G. performed the mouse xenograft experiments; H.P. performed bioinformatics analysis; H.P. and A.H. performed in vitro experiments with GBM 253; J.P., C.S. and H.G.-C. performed BTIC migration on nanopatterned surfaces; K.C.K., J.P. and R.R. wrote the manuscript, with edits from V.C.-G., H.G.-C. and A.Q.-H.; A.Q.-H. and R.R. provided funding and oversight for the project.

\section{Additional information}

Supplementary Information accompanies this paper at http://www.nature.com/ naturecommunications

Competing financial interests: The authors declare no competing financial interests

Reprints and permission information is available online at http://npg.nature.com/ reprintsandpermissions/

How to cite this article: Kondapalli, K. C. et al. A leak pathway for luminal protons in endosomes drives oncogenic signalling in glioblastoma. Nat. Commun. 6:6289 doi: $10.1038 /$ ncomms7289 (2015). 